\title{
Modular Organization of Occipito-Temporal Pathways: Cortical Connections between Visual Area 4 and Visual Area 2 and Posterior Inferotemporal Ventral Area in Macaque Monkeys
}

\author{
Daniel J. Felleman, Youping Xiao, and Evelyn McClendon \\ Department of Neurobiology and Anatomy, University of Texas Health Science Center-Houston Medical School, Houston, \\ Texas 77030
}

The modular organization of cortical pathways linking visual area 4 (V4) with occipital visual area 2 (V2) and inferotemporal posterior inferotemporal ventral area (PITV) was investigated through an analysis of the patterns of retrogradely labeled cell bodies after injections of tracers into V4 and PITv. Although cytochrome oxidase or other stains have failed to yield reliable independent anatomical markers for cortical modules beyond V1 and V2, V4 and PITv seem to have modular compartments with specific patterns of cortico-cortical connectivity. Tracer injections of V4 labeled cells in V2 (1) thin stripes exclusively, (2) interstripes exclusively, or (3) specific combinations of interstripe and thin stripe subcompartments. These labeling patterns suggest (1) that there is a complicated organization of inputs to V4, (2) that projections from V2 to V4 display a submodular selectivity, and (3) that projections from V2 to V4 display some degree of cross-stream convergence. Consistent with this framework, extensive regions of PITV provide feedback projections to interstripe-recipient portions of V4, whereas more restricted portions of PITv provide feedback to thin stripe-recipient portions of V4. Similarly, the feedforward projection from V4 to PITv often arose from multiple cell clusters across a wide expanse of V4. When distinguishable fluorescent tracers were injected into two PITv sites separated by 3-5 mm, a variety of projection patterns was observed in V4. In most cases, labeled cells were found in multiple, interdigitating, nonoverlapping clusters of 1-3 $\mathrm{mm}$ width, whereas in other cases the two labeled fields were highly intermixed. These results suggest that V4 and PITv contain functional modules that can be characterized by the specific patterns of segregated and convergent projections they receive from lower cortical areas. These specific patterns of intercortical input, in conjunction with intrinsic cortical circuitry, may endow extrastriate cortical neurons with new and more complex receptive field properties.

Key words: functional architecture; temporal lobe; cortical pathways; retrograde tracers; cytochrome oxidase
The supragranular layers of visual area 1 (V1) are characterized by cytochrome oxidase dense blobs surrounded by pale interblobs (for example, see Livingstone and Hubel, 1984), whereas V2 is characterized by alternating sets of thick and thin dense stripes separated by pale stripes (for example, see Hubel and Livingstone, 1987). Functional studies have contributed to the notion that V1 consists of three compartments associated with parallel functional streams: the blob-dominated stream (BD) involved in color analysis, the interblob-dominated stream (IB) involved in shape analysis, and the magnocellular-dominated stream (MD), which arises from layer $4 \mathrm{~B}$ and contains orientation and direction-selective cells that receive input from magnocellular LGN recipient layer 4C $\alpha$ (Van Essen and Gallant, 1994). These three functional streams target specific compartments in V2: the cytochrome oxi-

Received Sept. 3, 1996; revised Feb. 12, 1997; accepted Feb. 14, 1997.

Correspondence should be address to Dr. Daniel J. Felleman, Department of Neurobiology and Anatomy, University of Texas Houston Health Science Center, The Medical School, 6431 Fannin Street, Houston, TX 77030.

This work was supported by National Institutes of Health Grant EY-08372, National Institutes of Health Core Grant EY-10608, Texas Advanced Research Program Grant 011618-025, and a Grant-in-Aid in honor of Bob Hope of the Fight for Sight research division of Prevent Blindness America. Portions of this work were conducted while Dr. Felleman was an Alfred P. Sloan Fellow. We are grateful to Elizabeth Earle, Ki Lin, Rama Grenda, and Qian Huang for their excellent histology and section scoring. We also thank Rama Grenda for photographic and illustration assistance. We thank Andrzej Zych for his superior software support. We thank Dr. Ted DeYoe for developing and sharing the software for calculation of the SI in three-dimension anatomical section stacks. Finally, we thank Drs. Nikos Logothetis and John Maunsell for their critical comments on earlier drafts of this manuscript. Copyright (C) 1997 Society for Neuroscience $0270-6474 / 97 / 173185-16 \$ 05.00 / 0$ dase dense blobs project to V2 thin stripes, the V1 interblobs project to V2 interstripes, and layer $4 \mathrm{~B}$ projects to the V2 cytochrome oxidase thick stripes (Van Essen et al., 1986; Livingstone and Hubel, 1987).

Despite this apparent high degree of segregation of V1 inputs to V2, the degree to which V2 stripe compartments are functionally distinct has been brought into question. For example, DeYoe and Van Essen (1985) reported that V2 thin stripe and interstripes both contain chromatic selective neurons $(86 \%$ vs $64 \%$ ), and similar proportions of cells in thin stripes $(80 \%)$ and interstripes (83\%) were orientation selective. Similarly, Levitt et al. (1994) reported significant overlap in the response properties of neurons in V2 thin stripes and interstripes, although thin stripes tended to have lower spatial resolution and contrast sensitivity yet tended to have a lower incidence orientation selectivity. In contrast, optical recording studies of V2 functional architecture typically rely on differences in thin stripe and interstripe responses to isoluminant color and luminance contrast gratings. Most relevant, however, is the observation that $\mathrm{V} 2$ thin stripes are functionally heterogeneous, containing discrete regions of high color selectivity and luminance selectivity (Roe and Ts'o, 1995; Xiao and Felleman, 1996). This clustering of properties into submodules within cytochrome oxidase stripes may account for the reported overall similarities of properties of V2 thin stripes and interstripes.

The three cytochrome oxidase compartments of V2 have differential projections to other extrastriate cortical areas. Cells in 
the thick stripes project to middle temporal area (MT; DeYoe and Van Essen, 1985; Shipp and Zeki, 1985) and V3 (Felleman et al., 1988), whereas V4 is the major target of the thin and interstripe compartments (DeYoe and Van Essen, 1985; Shipp and Zeki, 1985, 1989; Nakamura et al., 1994). Given the high degree of modular segregation and cortico-cortical specificity in V1 and V2, it is important to determine whether this segregation persists into V4 and higher cortical areas. Alternatively, integration of these functional streams may take place at these intermediate levels of cortical processing.

DeYoe et al. $(1988,1994)$ used fluorescent retrograde tracers to demonstrate that two nearby loci in V4 can receive input from topographically overlapping but segregated populations restricted to V2 thin stripe or interstripe compartments. Zeki and Shipp (1989) suggested that this segregation is not complete in V4, but rather some foci receive input from interstripes only, whereas other foci receive a mixed thin stripe and interstripe input. Recently, optical recording of intrinsic cortical signals has shown that V2 stripe compartments are functionally heterogeneous; interstripes and thick stripes contain orientation-selective domains (Malach et al., 1994), and thin stripes consist of separate clusters of cells activated by chromatic and luminance stimuli (Roe and Ts'o, 1995). Furthermore, the pattern of cytochrome oxidase activity is nonuniform within dense stripes such that individual stripes are best described as a series of beads on a string rather than as a uniform structure. To what extent are these heterogeneities in V2 thin stripe cytochrome oxidase patterns and functional architecture reflected in the pattern of connections with V4?

V4 makes dense reciprocal connections with several subdivisions of inferotemporal cortex including posterior inferotemporal ventral area [(PITv, part of the temporal-occipital transition region (TEO)], central inferotemporal ventral area (CITv), posterior inferotemporal dorsal area (PITd), and central inferotemporal dorsal area (CITd; Van Essen et al., 1990; Distler et al.,1993). Whereas area TEO does receive a relatively weak input from both V2 thin stripe and interstripes (Nakamura et al., 1994), it has not yet been possible to determine whether local foci in TEO (or V4) receive input from V2 thin stripe or interstripe compartments (Nakamura et al., 1993). Furthermore, it has not yet been possible to relate the pattern of reciprocal feedback projections from PITv to V4 foci that have been characterized by their pattern of input from $\mathrm{V} 2$.

We describe here the modular and submodular organization of cortical pathways linking occipital and inferotemporal cortex. The results indicate that restricted V4 foci receive input from either $\mathrm{V} 2$ thin stripes or interstripes, some regions only receive input from submodular compartments within these stripes, whereas other loci receive convergent input from different submodular compartments. Multiple $\sim 2-3 \mathrm{~mm}$ wide clusters in V4 project to single foci in the PITv, whereas nearby foci receive input from segregated yet interdigitated V4 sites. These data suggest that modular segregation of cortical pathways is a widespread feature of cortical organization and is not limited to early levels of cortical processing. Portions of these data have been presented previously in abstract form (Felleman and McClendon, 1991a,b; Felleman et al., 1992) and in a short communication (DeYoe et al., 1994).

\section{MATERIALS AND METHODS}

Surgical methods and tracer injections. Experiments were carried out in 20 hemispheres of 15 juvenile or adult macaque monkeys (Macaca fascicularis, M. mulatta, or M. nemestrina). In preparation for surgery, the monkey was first premedicated with atropine $(0.04 \mathrm{mg} / \mathrm{kg}$, i.m.) and restrained with ketamine $(10-15 \mathrm{mg} / \mathrm{kg}$, i.m.) and later with Nembutal
(6-10 mg/kg, i.v.). An endotracheal tube was inserted, and the monkey was installed in a stereotaxic unit. Intraoperative anesthesia was provided by Halothane or Isofurane (1-2\%) and supplemented with Nembutal as needed. In one experiment (90F-L), a stainless steel recording chamber was mounted around a craniotomy to allow access to V4 and posterior inferotemporal cortex for microelectrode mapping experiments. In all other experiments, a craniotomy was performed to allow tracer injections into V4 and posterior IT, and was closed before recovery. In 10 cases the posterior half of the corpus callosum was cut, and in 8 cases HRP crystals and/or HRP-acrylamide gel was applied to the cut axons to label the pattern of interhemispheric connections in occipital and posterior inferotemporal cortex.

Tracer injections were made under visual guidance into V4 on the prelunate gyrus or into PITv, which was initially identified by its position anterior to the inferior occipital sulcus, in the vicinity of the posterior middle temporal sulcus (PMTS). In 6 of 10 cases, the injection into PITV was confirmed on the basis of its position anterior to the callosal band at the border between V4 and IT (see Felleman et al., 1996). Tracer injections included 200-300 $\mathrm{nl}$ bisbenzamide [BB, $10 \%$ in distilled water (DW); Sigma, St. Louis], nuclear yellow (NY, 4\% in DW; Sigma), fast blue (FB, 4\% in DW; Sigma), diamidino yellow (DY, 4\% in DW; Dr. Illing, Germany), wheat germ agglutinin (WGA)-HRP (1\% in Tris buffer, Sigma). Pressure injections were made using calibrated micropipettes using a time pressure injection system (WPI). Individual injections were made under visual guidance, and the injected volume was measured by the displacement of the meniscus within the calibrated pipette. After the last tracer injection, the dura was closed, the recording chamber or craniotomy was closed, and the monkey was allowed to recover for 2-7 d. Postoperative analgesia was provided by Buprenex $(0.01-0.03 \mathrm{mg} / \mathrm{kg}$ ) and was continued as needed.

At the termination of the anatomical tracer transport period, the monkey was deeply anesthetized with Nembutal (75-100 mg/kg, i.v.) and perfused intracardially with $0.1 \mathrm{M}$ phosphate buffer, $\mathrm{pH} 7.4$, followed by either $4 \%$ paraformaldehyde in phosphate buffer or $2.0 \%$ paraformaldehyde $/ 1.0 \%$ glutaraldehyde. In either case, the fixative was washed out with $0.1 \mathrm{M}$ phosphate buffer containing a graded series of sucrose $(0-20 \%)$ or glycerin $(0-10 \%)$. The brain was then removed, blocked, and photographed before sectioning.

In 12 cases, the perfusion was brief $(8-10 \mathrm{~min})$ to permit cortical unfolding before sectioning. In these cases, the brain was removed from the skull, and the occipital operculum was removed by cutting along the fundus of the lunate sulcus and inferior occipital sulcus, and across the stem of the calcarine sulcus. This block of tissue was then gently pressed between glass slides, briefly post-fixed in the original cryoprotective fixative, and later sectioned tangential to the pial surface. The prelunate gyrus and posterior temporal cortex were similarly unfolded and flattened using blunt dissection of the underlying white matter (Olavarria and Van Sluyters, 1985; Tootell and Silverman, 1985). In one case, the superior temporal sulcus was not unfolded from the bulk of the inferior temporal lobe, and the whole block of tissue was sectioned tangentially. In the remainder of cases, utilizing unfolded blocks of V4-PITv, the lateral bank of the superior temporal sulcus was unfolded to allow tangential sectioning of a large block of anterior occipital and inferior temporal cortex. This cortical unfolding process is summarized schematically in Figure 1.

Tissue processing and data analysis. Frozen sections were cut on a sliding microtome at $31 \mu \mathrm{m}$ for horizontal material and at $50 \mu \mathrm{m}$ for tangential material. Sections were collected in phosphate buffer $(0.1 \mathrm{M})$ and were processed immediately for HRP using the TMB method of Mesulam et al. (1978; see Felleman et al., 1996). Sections containing fluorescent, retrogradely labeled cells were mounted on glass slides from $0.05 \mathrm{M}$ phosphate buffer and air dried before observation under UV epifluorescence using a Zeiss Axiophot microscope. Alternative sections were processed for cytochrome oxidase histochemistry according to the protocol of Wong-Riley and Carroll (1984). The myeloarchitectonic borders of V3, MT, and floor of the superior temporal sulcus area (FST) were determined from sections stained according to the protocol of Gallyas (1979) in 5 of 10 PITv injection cases.

A computer-interfaced microscope system (Neurolucida) was used to record the positions of labeled cells, axon terminals, borders of cytochrome oxidase compartments, as well as blood vessels, injection site, and section edge lines. These data allowed for the precise encoding of cell or terminal position and thus the cortical layers of pathway origin or termination. These data were then exported to a Silicon Graphics workstation (Personal Iris 4D/20G, Indigo ${ }^{2} \mathrm{XL}$, or Indigo ${ }^{2}$ High Impact) where custom software allowed for the reconstruction of the three-dimensional rela- 

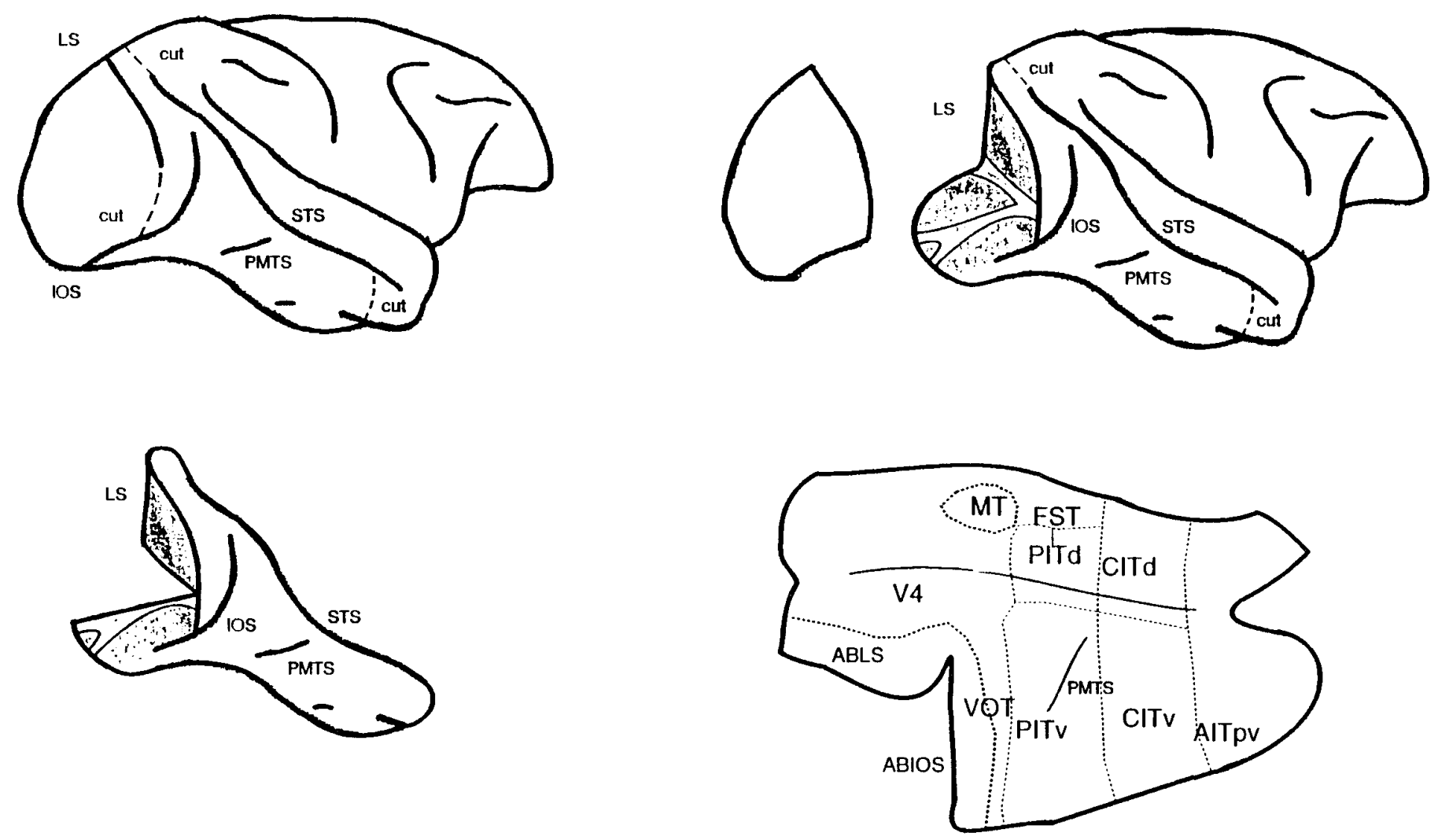

Figure 1. Occipito-temporal unfolding. The occipital operculum is removed by cutting the cortex at the fundus of the lunate and inferior occipital sulci. The cut is then extended through the parieto-occipital sulcus and across the stem of the calcarine sulcus and occipito-temporal sulcus. The anterior occipital and posterior two-thirds of the temporal lobe are then separated from the remainder of the brain through cuts made at the primary fundus of the STS and in the OTS. The white matter underlying the STS and prelunate gyrus is then undercut, and the block of tissue is gently unfolded. Frozen sections were then cut at $50 \mu \mathrm{m}$ on a large platform microtome. $A B L S$, Anterior bank of the lunate sulcus; $L S$, lunate sulcus.

tionships among labeled structures (ANATOMY, Caltech, Division of Biology and later modifications at the University of Texas Medical School). In tangential reconstructions, radially aligned blood vessels provided an important additional source of information to guide the three-dimensional alignment of adjacent or nearby tissue sections. These tangential cases provided an important means to visualize the detailed horizontal distribution of labeled cells. Although each three-dimensional stack contains only 4-8 sections, the number of labeled cells scored in each section is typically several thousand. The horizontally sectioned material was used to study the detailed laminar distribution of labeled cells and to provide a means for measuring the size of individual cell clusters. Pairs or trios of such sections were used for quantitative analysis of cell segregation or intermixing according to a segregation index (DeYoe et al., 1994).

The distribution of any single label type (HRP, fluorescent retrograde tracers, cytochrome oxidase) was analyzed in a series of sections spaced between $125(1: 4$ sections at $31 \mu \mathrm{m})$ and $200(1: 4$ sections @ $50 \mu \mathrm{m}) \mu \mathrm{m}$ apart. In V2, cytochrome oxidase-dense regions were outlined in 3-4 sections, and these patches were reconstructed into stripes. Cytochrome oxidase patterns considered useful to excellent were visualized after nine injections into five hemispheres. In these cases, the three-dimensional workstation was used to align the pattern of retrogradely labeled cells with high resolution images of cytochrome oxidase sections to determine the compartment of their origin. In the remaining four hemispheres, technical limitations prevented adequate cytochrome oxidase histochemistry. After these seven injections, retrogradely labeled cells were assigned to V2 compartments on the basis of the overall pattern of labeling and distances between labeled stripes. In these cases, a separation of 4 $\mathrm{mm}$ implies labeling of V2 thin stripes, whereas "doublet" patterns with $2 \mathrm{~mm}$ separations imply labeling of V2 interstripes (DeYoe et al., 1988, 1994; Shipp and Zeki, 1991). In tangential sections, data from infragranular and supragranular labeling were combined because of the imperfect unfolding of the large cortical sheet. Although cytochrome oxidase histochemistry has not revealed a modular organization in V4 (but see
Tootell et al., 1994), the differences in laminar patterns allowed for a rapid assessment of the degree of cortical flattening in this material.

Two-dimensional cortical maps were used to represent a variety of anatomical data including the pattern of interhemispheric connections (eight cases); myeloarchitectonic borders of V3, MT, and FST (six cases); the distribution of labeled cells; as well as the sulcal borders of individual brains. This method allows for a compact representation of a large number of data types and thus facilitates the analysis of the interrelationships among labeling patterns.

The distributions of labeled cells in V2, V4, and PITv were analyzed from three-dimensional reconstructions of tangential or horizontal brain sections. The relative segregation or intermixing of two populations of labeled cells was quantified using a segregation index (SI; DeYoe et al., 1994). First, a three-dimensional reconstruction of the area of interest is performed using customized software on a Silicon Graphics Workstation $\left(4 \mathrm{D} / 20 \mathrm{G}\right.$ or Indigo $\left.{ }^{2} \mathrm{XL}\right)$. Second, a region of interest is isolated (a series of horizontal sections spanning $0.5-2 \mathrm{~mm}$, or a full cortical thickness in a tangential series) and only one label type is displayed. Third, counting boxes are placed surrounding each cluster of labeled cells as well as the whole region of interest. Fourth, this procedure is repeated for the other label types. Next, the distribution index $\left(D_{i}\right)$ was calculated as: $D_{i}=A_{i}-$ $\mathrm{B}_{\mathrm{i}} / \mathrm{A}_{\mathrm{i}}+\mathrm{B}_{\mathrm{i}} ; \mathrm{D}_{\text {tot }}=\mathrm{A}_{\text {tot }}-\mathrm{B}_{\text {tot }} / \mathrm{A}_{\text {tot }}+\mathrm{B}_{\text {tot }}$. Finally, a normalized SI is computed: SI $=\left|D_{\mathrm{i}}-\mathrm{D}_{\text {tot }}\right|$ that reflects the average segregation within each cluster of cells normalized for the overall number of cells labeled by each tracer in each projection field. Whereas attempts were made to inject equal volumes of tracer $(250 \mathrm{nl})$ at each injection site, differences in tracer sensitivity, precise laminar position of the injection sites, and other uncontrolled factors make quantitative comparisons of the size of projection fields or density of labeled pathways difficult.

Identification of cortical areas. A variety of criteria was used to identify the various cortical areas that were injected or labeled in the current experiments. V1 was identified by its unique cytoarchitecture and position in the brain. V2 was identified as a belt of cortex that is generally immediately adjacent to V1. The characteristic patterns of staining by 


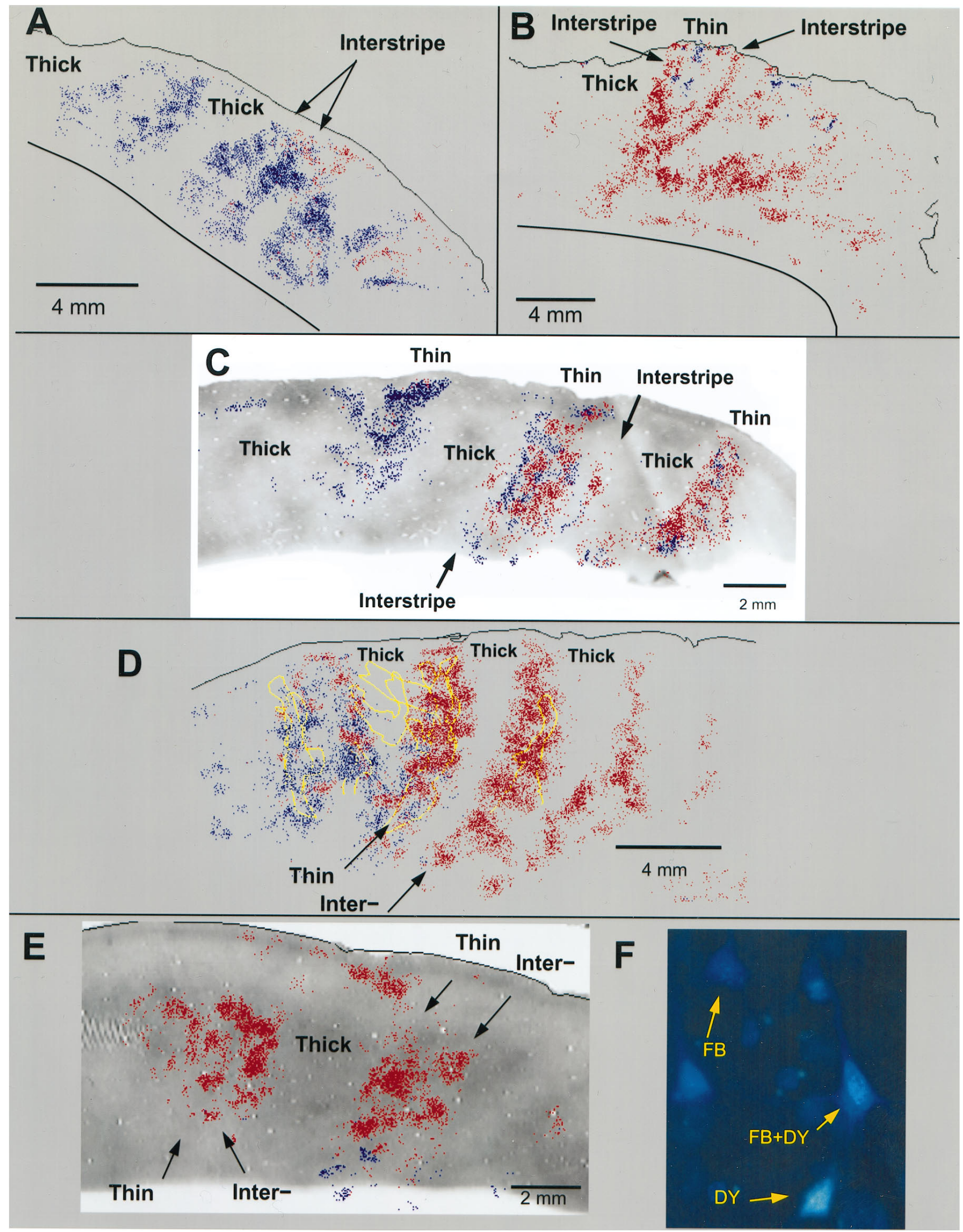

Figure 2. V2 Projections to V4. A, Projections from V2 interstripe doublets. BB-labeled cells (blue) are distributed in three sets of doublets corresponding to interstripes. Several stripes consist of chains of dense cell clusters separated by cell sparse gaps. NY-labeled cells (red) are scattered in thin stripe and interstripe zones (case 92-2L). B, Segregated projections from V2 thin and interstripes to V4. NY-labeled cells were found in V2 interstripes, whereas weak clusters of BB-labeled cells were found in three cytochrome oxidase thin stripes. Cytochrome oxidase architecture become rather complex and stripes curve significantly in this region of foveal V2 (case 91-5R). C, Heterogeneous V2 thin stripe (Figure legend continues) 
cytochrome oxidase histochemistry was also used to identify V1 and V2. V3 was identified as a belt of densely myelinated cortex immediately anterior to dorsal V2. V3, and the ventrally positioned VP, are located immediately posterior to dense callosal bands that indicate the location of the vertical meridian. V3A was identified as a callosal-free ring within dorsal cortex of the prelunate gyrus, anterior to V3 and posteromedial to V4. Lower field portions of V4 are contained in dorsal cortex of the prelunate gyrus and upper field V4 is located in ventral cortex on the anterior bank of the inferior occipital sulcus. In dorsal cortex, V3A is separated from V4 by a dense band of callosal projections. In ventral cortex, this band corresponds to the border between VP and V4. The anterior border of VOT is coincident with an anterior band of callosal projections at the border with PITv. The border between V4 and VOT is contained within the ventral callosal-free zone but is currently not distinguishable using conventional architectonic methods. V4t is a narrow belt of cortex that forms the lateral border of MT. At the present time, the dorsal occipito-temporal area (DOT) is identified as a lower field V4 projection zone located immediately anterior to V4 near the tip of the inferior occipital sulcus (IOS) and extending into the superior temporal sulcus (STS). It remains to be determined to what extent V4t and V4 or DOT and V4t form common borders. DOT may contain a compressed representation of the lower field and may not extend dorsally along the lateral border of V4t. Thus, at peripheral eccentricities, V4 may adjoin $\mathrm{V} 4 \mathrm{t}$, whereas in central vision, DOT may be located along the anterior border of V4. PITV is identified as a major V4 projection zone in posterior inferotemporal cortex, anterior to DOT and VOT. PITV is generally located immediately posterior to the PMTS, which often identifies the border between PITv and CITv. The lower visual field is represented anterior to upper field in PITv, with peripheral eccentricities represented in more ventral cortex.

\section{RESULTS}

The overall goal of the current experiments was to determine the compartmental organization of feedforward and feedback pathways that link V4 with V2 and PITV. In two different series of experiments, tracer injections in V4 or PITv were used to (1) identify the compartmental and subcompartmental organization of V2 inputs to $\mathrm{V} 4,(2)$ determine the organization of feedback projections that link inferotemporal cortical areas with characterized foci in V4, and (3) determine the organization of V4 outputs to inferotemporal cortical PITv.

\section{Organization of V2 inputs to V4}

To describe the specific compartmental and subcompartmental organization of feedforward inputs from V2, two single and seven paired injections of distinguishable retrograde tracers were made at random locations within foveal and parafoveal V4. Robust labeling of V2 was observed in 13 cases (13 of 16; 81\%), whereas weak labeling limited the analysis of several injections ( 3 of 16 ; $19 \%$ ). A variety of different patterns of V2 input was observed after injections into individual V4 loci. In $62 \%$ of cases ( 8 of 13), labeled cells were found primarily either in thin stripes (4 of 13; $31 \%$ ) or in interstripes ( 4 of $13 ; 31 \%$ ). Labeled cells were found to cross thin stripe and interstripe compartments in more than one-third of the cases ( 5 of $13 ; 38 \%$ ).

\section{Projections to V4 from V2 thin or interstripe compartments}

After approximately one-half of the V4 injections, labeled cells in V2 were found largely confined to either thin stripes (4 of 16; $25 \%$ ) or interstripes ( 4 of $16 ; 25 \%$ ). Figure $2 A$ illustrates the distribution of retrogradely labeled cells in V2 after paired injections in V4 on the prelunate gyrus near the tip of the IOS (see Fig. $3 A$ ). In V2, BB-labeled cells were found in three doublets that were separated by approximately $4 \mathrm{~mm}$ and spanned nearly 10 $\mathrm{mm}$ of opercular V2. The individual labeled stripes were approximately $1 \mathrm{~mm}$ wide and seemed to consist of multiple $\sim 1 \mathrm{~mm}$ wide cell clusters. Each doublet was separated by a gap that ranged in width from less than $500 \mu \mathrm{m}$ to more than $1 \mathrm{~mm}$. Because individual cytochrome oxidase thin stripes are approximately 1 mm wide, the observed labeling pattern is consistent with dense interstripe input and no input from the center of thin stripes. Nuclear yellow-labeled cells were found in partial topographic overlap with two of the three sets of BB-labeled stripes. In the region of overlap, the NY-labeled cells were largely segregated from the BB-labeled cells in a pattern that is suggestive of thin stripe compartments.

A similar pattern of segregated interstripe and thin stripe projections was observed in case 2 after paired $\mathrm{BB}$ and $\mathrm{NY}$ injections in V4 at the tip of the IOS (see Fig. $3 B$ ). In V2, BB-labeled cells were found in two small clusters that aligned into a narrow stripe surrounded by dense doublet stripes of NYlabeled cells. The periodicity of this pattern and its correspondence with the cytochrome oxidase pattern indicates that the BB-labeled cells are located within V2 thin stripes, whereas the NY-labeled cells are located primarily within V2 interstripes.

\section{Projections to V4 from V2 thin stripe subcompartments}

In two cases of dual V4 injections ( 2 of $16 ; 12 \%$ ), labeled cells were confined largely to V2 thin stripes, but separate arrays of interdigitating cell clusters were observed to project to the separate V4 injection sites. In case 3, small injections of BB and NY were made into V4 just dorsal to the tip of the IOS, as illustrated in the reconstruction of the unfolded occipito-temporal cortex in Figure $3 C$. Labeled cells in V2 were distributed into 3 distinct stripe-like compartments in mid portions of the operculum (Fig. 2C). These labeled cells were identified as thin stripes because of their close association with alternate cytochrome oxidase-dense stripes visualized from adjacent sections. These labeled stripes were separated by $\sim 4 \mathrm{~mm}$, reinforcing the view that the observed cytochrome oxidase pattern was complete and that alternative (thin) stripes were labeled by the V4 injections. Close inspection of the relationship between the labeled cells and the cytochrome oxidase image indicates that the majority of labeled cells overlies the dense thin stripe, whereas a few clusters of NY-labeled cells were separated from this main cluster and were centered in an interstripe zone. This is most clearly ob-

\section{$\leftarrow$}

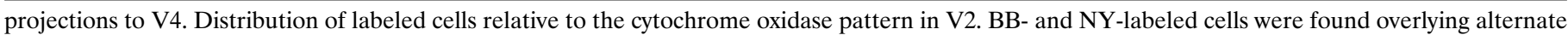

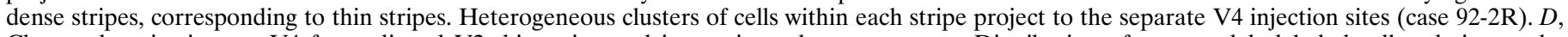

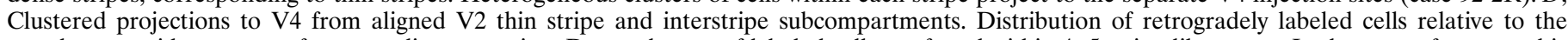

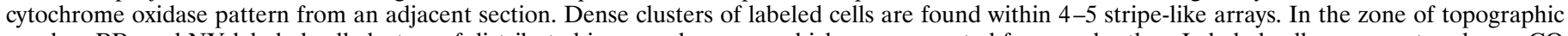

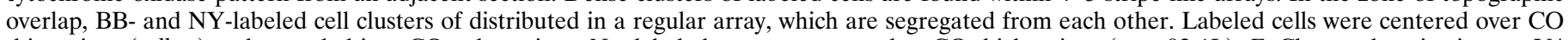

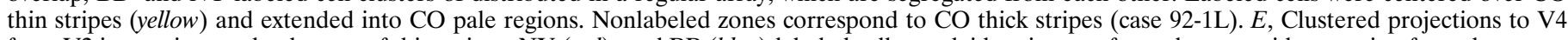

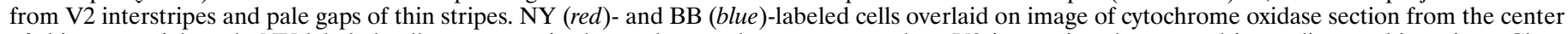

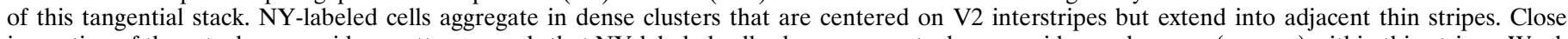

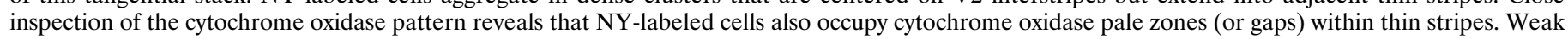

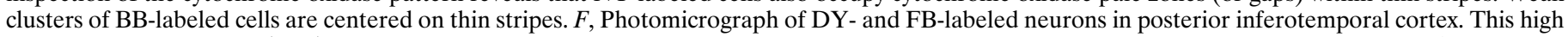

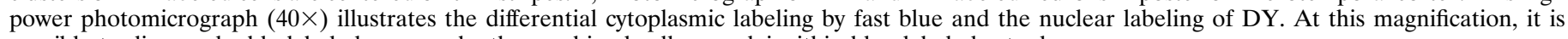
possible to discern double labeled neurons by the combined yellow nuclei within blue-labeled cytoplasm. 

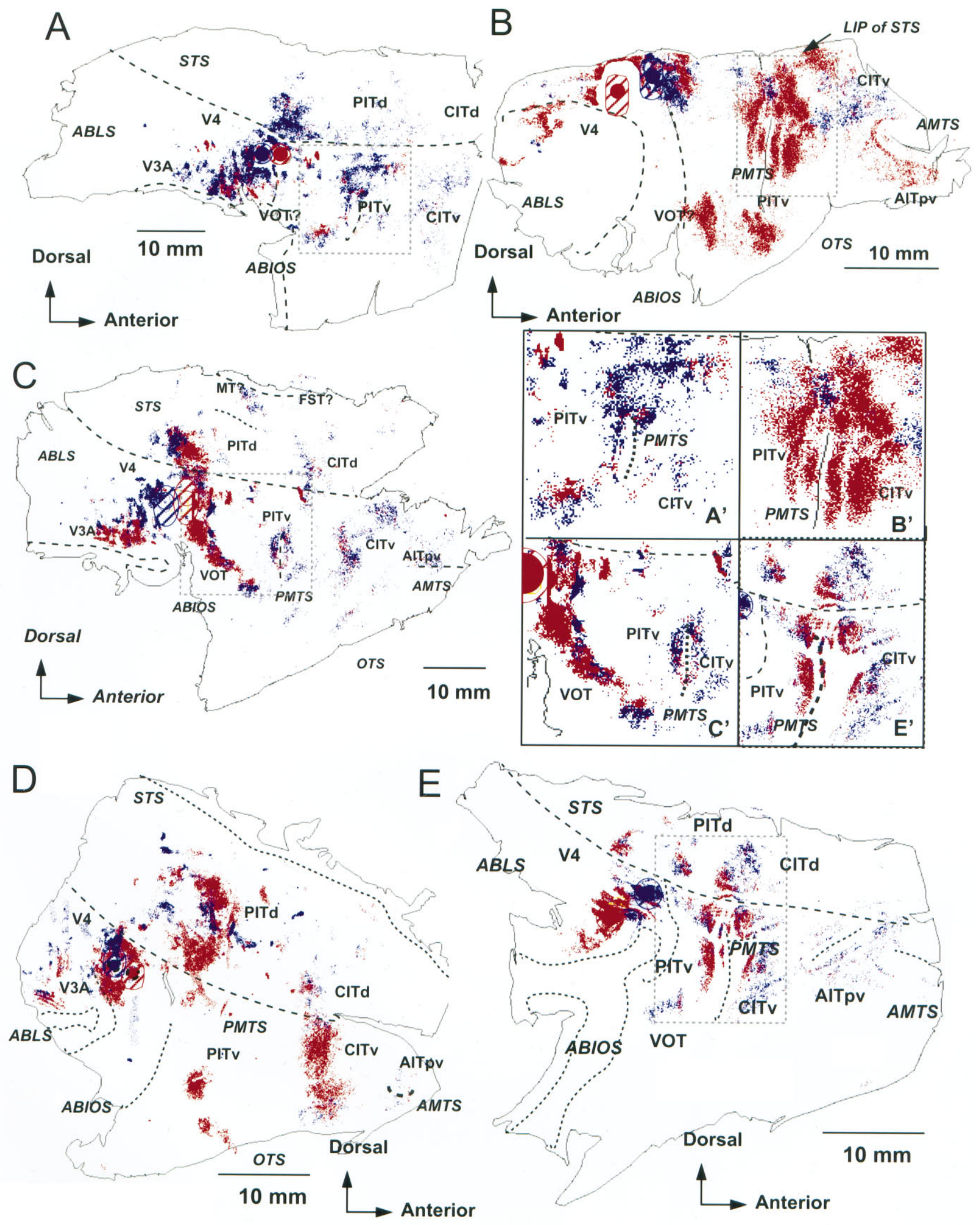

Figure 3. Feedback projections to V4 from occipito-temporal cortex. A, Connections of interstripe recipient portions of V4. BB (blue) and NY (red) injection sites in parafoveal portions of V4 on the prelunate gyrus. Dense clusters of BB-labeled cells were found surrounding the PMTS in PITv. Weaker projections were observed in more anterior cortex corresponding to CITv and in PITd within the STS. Inset shown at higher magnification in $A^{\prime}$. $B$, Connections of thin stripe and interstripe recipient portions of V4. BB (blue) and NY (yellow) injection sites in foveal V4 on the prelunate gyrus in unfolded occipito-temporal cortex. Dense fields of NY-labeled cells were found surrounding the PMTS (PITv), which extended (Figure legend continues) 
served for the central labeled stripes, but also is obvious in the more lateral stripe as well.

The association between dense cytochrome oxidase clusters and $\mathrm{BB}$ projections is most clear in the peripheral-most stripe (at left). In this region, BB-labeled cells are in nearly precise register with the cytochrome oxidase-dense clusters of this thin stripe such that cytochrome oxidase-dense regions are heavily labeled whereas cytochrome oxidase-pale regions are largely unlabeled.

In this case, each retrograde tracer injection labeled distinct clusters of cells approximately $500-1000 \mu \mathrm{m}$ in width. Each V2 stripe seems to be made up of a large number of these cell clusters ( 10 or more clusters for each of 2 labels; 20 clusters total). A total of three stripes containing as many as 20-30 cell clusters was labeled by this pair of injections. Cell densities ranged from 10 to 400 cells $/ \mathrm{mm}^{2}$. The pattern of labeling is consistent with two distinct processing modules within $\mathrm{V} 2$ thin stripes that may reflect segregated processing of different colors or may reflect the segregated processing of chromatic and luminance cues by thin stripes (see Discussion).

\section{Projections to V4 from aligned V2 thin stripe and interstripe subcompartments}

In four cases of paired fluorescent retrograde tracer injections of V4, clusters of retrogradely labeled cells straddled thin stripe and interstripe compartments. In case 4 , dense clusters of retrogradely labeled cells formed elongated stripes across the width of V2. Figure $3 D$ illustrates the locations of the BB and NY injection sites in V4, separated by approximately $4 \mathrm{~mm}$ on the prelunate gyrus just dorsal to the tip of the IOS. Figure $2 D$ illustrates the distribution of retrogradely labeled cells in a tangential section of opercular V2. Although the quality of the cytochrome oxidase reaction in this block was poor, it was possible to discern a series of dense cytochrome oxidase stripes running roughly perpendicular to the edge. The narrow, dense bands of increased $\mathrm{CO}$ activity were separated by variable sized pale zones containing weaker bands of increased cytochrome density. The dense CO bands were separated by approximately $3.5-4.0 \mathrm{~mm}$ and thus were likely to reflect a full cycle of V2 compartments rather than individual thin and thick stripes. Thus, V2 thick stripes were largely cytochrome pale in this hemisphere.

It was possible to align several of the elongated projection fields with dense cytochrome oxidase compartments. In this experiment, clusters of NY labeled cells extend along the full width of 4 adjacent thin/inter stripes. The NY-labeled clusters clearly extended contiguously across the thin stripe into the interstripe compartment on either side. A remarkable alternating pattern of BB- and NY-labeled cell clusters was observed along the left margin of the second stripe from the left. In this stripe, individual BB- or NY-labeled cell clusters were approximately $1 \mathrm{~mm}$ across and the gaps in the pattern of NY-labeled cells were filled in by
BB-labeled cells. In this hemisphere, a total of 20 cell clusters was observed with densities ranging from 10 to 185 cells $/ \mathrm{mm}^{2}$.

In case 5, dense clusters of NY-labeled cells were found to cross between thin stripe and interstripe compartments; however, only portions of the thin stripes or interstripes were labeled. The locations of the injection sites in parafoveal V4 of the prelunate gyrus are illustrated in Figure $3 E$. The distribution of labeled cells with respect to the pattern of cytochrome oxidase activity in V2 is illustrated in Figure $2 E$. In this case, multiple dense clusters of labeled cells were found within the pale interstripe regions surrounding two cytochrome-dense thin stripes. However, additional clusters of labeled cells were found within the adjacent thin stripes centered on the pale gaps within the stripes. This pattern of labeling is consistent with single V4 loci receiving convergent input from subpopulations of cells located in thin stripes and interstripes of V2. Because these cells were located in V2 thin stripe "pale" zones, such a pattern of labeling would be consistent with a convergence of nonoriented luminance processing with orientation specific luminance processing in V4. Alternatively, the observed pattern of labeling could result from tracer injections that fell at the border between specific thin stripe and interstripe subcompartments.

\section{Summary of $V 2$ projections to V4}

The observed results suggest that cell clusters in V2 make several types of connections with V4. First, injections of V4 can label cells located predominantly in either thin stripes or interstripes, thus indicating a compartmental segregation of V2 projections to portions of V4. Second, injections of V4 can label multiple interdigitating yet segregated cell clusters within individual thin stripes. This indicates that some portions of V4 receive input from specific subcompartments within V2 thin stripes, whereas other V4 foci receive input from adjacent V2 subcompartments. Finally, injections of V4 can label submodular projection clusters that extend across V2 thin stripe and interstripe compartment boundaries. These findings suggests that portions of V4 receive inputs arising from selective portions of both stripe compartments.

\section{Organization of inferotemporal feedback projections to V4}

Overview

The pattern of feedback projections to V4 from the temporal lobe was examined after the same injections of retrograde tracers in V4 used to analyze V2 connections. The compartmental organization of the inputs from V2 characterized these V4 foci as receiving (1) thin stripe input, (2) interstripe input, (3) submodular thin or interstripe input, or (4) mixed thin stripe and interstripe input. The single and paired V4 injections described above labeled numerous subdivisions of ventral occipital and inferotemporal cortex. The clustered projections from posterior IT subdivisions

$\leftarrow$

into more anterior cortex (CITv). Weaker projections were found from antero-ventral cortex (AITpv). BB-labeled cells were found in several weak clusters near the tip of the PMTS at the crown of the STS (PITv) and in more anterior cortex (CITv). Additional projections to PITd, CITd, and several other fields are not illustrated. Inset shown at higher magnification in $B^{\prime}$ (case 91-5). $C$, Connections of a thin stripe recipient portion of V4. Locations of injection sites in V4 on the prelunate gyrus. A dense band of labeled cells extended across the occipito-temporal junction from within the STS to below the PMTS. Segregated clusters of BB- and NY-labeled cells are found within this band particularly within VOT. Segregated clusters of labeled cells were found across inferotemporal PITv, CITd, CITv, and AITpv. Inset shown at higher magnification in $C^{\prime}$ (case 92-2R). D, Connections of a V2 thin interstripe convergent domain of V4. BB and NY injections in V4 on the prelunate gyrus. Retrogradely labeled cells were found in dense projection fields within the STS (PITd and CITd) and within the middle and inferior temporal gyri (PITv and CITv). Weaker BB projection fields were segregated from the NY-labeled cell clusters (case 92-1L). E, Connections of an interstripe-thin stripe convergence domain in V4. BB and NY injections sites on the prelunate gyrus in parafoveal V4. Dense clusters of NY-labeled cells were found in PITv and CITv. Weaker labeling extended into PITd within the STS and to AITpv. Inset shown at higher magnification in $E^{\prime}$ (case 92-4R). 
suggests that $\mathrm{V} 4$ loci that receive $\mathrm{V} 2$ thin stripe input receive a restricted feedback projection from PITv, whereas V4 loci that receive V2 interstripe inputs receive a more extensive inferotemporal input.

\section{Temporal lobe projections to V2 thin stripe and interstripe recipient zones of $V 4$}

The pattern of feedback projections that arise from occipitotemporal cortex after tracer injections into segregated V2 interstripe and thin stripe-recipient portions of $\mathrm{V} 4$ is illustrated in Figure $3, A$ and $B$. In Figure $3 A$, dense clusters of BB-labeled cells, associated with interstripe labeling, were found across a large number of inferotemporal subdivisions. In PITv, near the anterior limb of the PMTS, labeled cells extended over more than $20 \mathrm{~mm}^{2}$. This region of inferotemporal cortex (dashed box region) is illustrated at higher magnification in the inset labeled $A^{\prime}$. In this hemisphere, smaller clusters of NY-labeled cells (red), associated with thin stripe labeling in V2, were observed in PITv and perhaps in the ventral occipito-temporal area (VOT) on the anterior bank of the inferior occipital sulcus (ABIOS).

Another example of the segregated pattern of feedback labeling of the temporal lobe after injections of V4 loci that receive segregated thin stripe and interstripe input is illustrated in Figure $3 B$ and insert $B$ ' (see also DeYoe et al., 1994). In this experimental hemisphere, a large number of NY-labeled cells, associated with V2 interstripe labeling, were found throughout a large region surrounding the PMTS. Additional projection fields were located laterally: the first on the anterior bank of the IOS that may represent a projection from V4 or VOT, and a second field near the lateral bank of the occipito-temporal sulcus (OTS) that may represent a modular projection field within PITv, or a projection from a distinct area such as VTF. Several clusters of BB-labeled cells, associated with V2 thin stripe labeling, were found surrounded by the massive clusters of NY-labeled cells in PITV and more anterior cortex including CITv. These results suggest that $\mathrm{V} 4$ foci that receive input from V2 interstripes are associated with multiple large domains within PITv, whereas V4 thin striprecipient foci are associated with smaller, segregated domains within the temporal lobe.

\section{Temporal lobe projections to V2 thin stripe subcompartment recipient zones of V4}

The locations of the injection sites and the distribution of labeled cells in a computer reconstruction of unfolded anterior occipital and inferotemporal cortex are illustrated in Figure $3 C$ and inset $C$ '. These injections labeled numerous alternating clumps of BB- and NYlabeled cells stretching from the anterior bank of the IOS, onto the exposed cortex just anterior to the tip of the IOS, and halfway down the lateral bank of the STS. In this experimental hemisphere, the corpus callosum was not cut, so the borders between most areas cannot be made with precision. However, the additional clusters of labeled cells approximately $5 \mathrm{~mm}$ anterior to the IOS band are likely to represent weak labeling of PITv. Therefore, the dense band of labeled cells in this hemisphere may represent (1) an exquisite pattern of intrinsic circuitry in V4; (2) projections arising from VOT and DOT, immediately anterior to V4 in ventral and dorsal cortex, respectively; or (3) projections arising from PITv and PITd that are located in unusually posterior positions in IT. The absence of callosal label makes it difficult to distinguish among these possibilities, but the third seems unlikely given the other labeling patterns in IT. Therefore, it remains unclear whether the observed pattern is intrinsic or extrinsic to V4.
Retrogradely labeled cells were also found in additional projection fields in central and near anterior portions of IT. In general, BB- and NY-labeled cells were intermixed in these central and anterior IT fields. However, the projection field in central IT designated CITv contains three large clusters of NY-labeled cells in a larger field of BB-labeled cells.

\section{Temporal lobe projections to V2 thin stripe and interstripe subcompartment convergent zones of V4}

The distribution of labeled cells in anterior occipital and posterior to mid inferior temporal cortex from case 4 is illustrated in a reconstruction of unfolded, tangentially sectioned cortex in Figure $3 D$. Similar to the distribution of label in V2, the majority of labeled cells in IT cortex were labeled by the NY injection. BB-labeled cells were usually found intermixed with NY-labeled cells, although segregated BB-labeled clusters were observed in the STS and central portions of IT. The NY injection into V4 at the tip of the IOS that received input from V2 interstripes, labeled multiple clusters of cells anterior to the injection site. Two major patches were located immediately anterior on the middle temporal gyrus, and two other prominent patches were located in medial cortex within the OTS. Another field was observed near the posterior end of the anterior middle temporal sulcus, whereas a smaller, more diffuse focus was seen within the STS medial to CITv. Finally a cluster of labeled foci was found medial to the injection site within the STS. This cluster of projections undoubtedly includes V4t, FST, as well as intrinsic connections within V4. In summary, this interstripe-thin stripe convergence zone of V4 also receives feedback inputs from large clusters of cells distributed across several distinct areas within IT. Whereas the NYlabeled cells predominated, distinct clusters of BB-labeled cells were found in PITd, CITd, and CITv. Therefore, the microsegregated pattern of labeling observed to cross from V2 thin stripes into interstripes, is reflected in the organization of feedback projections from the several foci temporal lobe.

The distribution of labeled cells in anterior occipital to central inferotemporal cortex from case 5 is illustrated in Figure $3 E$ and insert $E^{\prime}$. Whereas the pattern of labeling in V2 was heavily biased in favor of NY-labeled cells, the projections from anterior occipital and inferotemporal cortex were much more balanced. Several clusters of labeled cells were found immediately anterior to the injection site, both within the STS and exposed inferior temporal gyrus. Three or more dense cell clusters were found more anteriorly in PITv, just posterior to the PMTS. BB-labeled cells were found in several small but dense clusters in PITv. The majority of labeled cells in PITv were NY labeled by the V4 injection that received a combination of specific thin stripe and interstripe submodular input. Anterior to the PMTS, BB- and NY-labeled cells were more equally balanced in number and areal extent of labeled clusters (see Figure 3, insert $D^{\prime}$ ). Anterior to this projection zone, a weaker field of intermixed BB- and NY-labeled cells was observed immediately posterior to the AMTS in the anterior inferotemporal posterior ventral area (AITpv).

\section{Summary of V2 and inferotemporal inputs to V4}

The compartmental organization of V2 outputs and the pattern of temporal lobe projections to V4 observed in these experiments is summarized in Table 1. The results indicate that individual foci in $\mathrm{V} 4$ receive input from thin stripes, interstripes, portions of thin stripes, portions of interstripes, or portions of both thin stripes and interstripes. The observation that submodular compartments within thin or interstripes can be labeled from V4 suggests that V4 


\begin{tabular}{|c|c|c|c|c|c|c|c|c|c|c|c|}
\hline $\begin{array}{l}\text { Case \# } \\
\text { tracer }\end{array}$ & V2 Stripe(s) & $\begin{array}{l}\text { No. of } \\
\text { stripes }\end{array}$ & Width & Separation & $\mathrm{CO}$ & SI & VOT/DOT & PITv & PITd & CITv & CITd \\
\hline $1-\mathrm{FB}$ & Thin & 3 & 0.5 & 4 & + & 1.72 & - & $\mathrm{C}$ & $?$ & $\mathrm{D}$ & $?$ \\
\hline 1-DY & Inter & 4 & 1 & $2-3$ & + & 1.72 & $\mathrm{D}$ & $\mathrm{D}$ & $?$ & $\mathrm{~S}+\mathrm{C}$ & $?$ \\
\hline 2-BB & Thin & 3 & $1-1.5$ & $2-5$ & - & - & $\mathrm{D}$ & $\mathrm{D}$ & $\mathrm{W}$ & $\mathrm{D}$ & $\mathrm{W}$ \\
\hline $2-\mathrm{NY}$ & Scattered & 1 & $\sim 0.5$ & - & - & - & $\mathrm{C}$ & $\mathrm{C}$ & S & - & - \\
\hline $3-B B$ & Inter & 4 & $\sim 1$ & 2 & - & $\mathrm{T}$ & $\mathrm{D}$ & $\mathrm{D}$ & S & S & $\mathrm{W}$ \\
\hline $3-\mathrm{NY}$ & Thin + inter & 3 & 1 & 2 & - & $\mathrm{T}$ & - & - & - & - & - \\
\hline $4-B B$ & Inter + thin & 3 & 2 & 4 & + & 1.41 & $\mathrm{D}$ & $\mathrm{W}$ & $\mathrm{S}$ & $\mathrm{S}$ & $\mathrm{C}$ \\
\hline 4-NY & Thin + inter & 5 & $1-2$ & 4 & + & 1.41 & $\mathrm{D}$ & $\mathrm{D}$ & S & $\mathrm{D}$ & $\mathrm{C}$ \\
\hline $5-\mathrm{BB}$ & Thin & 3 & 2 & 4 & ++ & 0.58 & $\mathrm{C}$ & DP & S & $\mathrm{D}$ & $\mathrm{D}$ \\
\hline $5-\mathrm{NY}$ & Thin & 3 & 2 & 4 & ++ & 0.58 & $\mathrm{C}$ & DP & $\mathrm{S}$ & $\mathrm{D}$ & $\mathrm{W}$ \\
\hline 6-BB & Inter & 6 & 1 & $2 ; 3$ & - & $\mathrm{T}$ & $\mathrm{C}$ & $\mathrm{D}$ & $\mathrm{W}$ & $\mathrm{W}$ & S \\
\hline $6-\mathrm{NY}$ & Thin (w) & 3 & 1 & 2 & - & $\mathrm{T}$ & $\mathrm{C}$ & $\mathrm{C}$ & S & S & - \\
\hline $7-\mathrm{NY}$ & Thin + inter & 2 & $2.5(\max )$ & 2.5 & ++ & - & $\mathrm{C}$ & $\mathrm{D}$ & $\mathrm{C}$ & $\mathrm{C}$ & $\mathrm{W}$ \\
\hline 7-BB & Thin $(w) ?$ & $1+$ & 0.5 & 2 & ++ & - & $\mathrm{C}$ & WC & $\mathrm{D}$ & $\mathrm{D}$ & S \\
\hline 8-HRP & Thin-inter & 3 & 2 & 2 & - & - & - & $\mathrm{D}$ & $\mathrm{D}$ & $\mathrm{W}$ & $\mathrm{W}$ \\
\hline 9-HRP & Inter & 2 & 1 & 2 & ++ & - & - & $\mathrm{C}$ & WC & $\mathrm{C}$ & WC \\
\hline
\end{tabular}

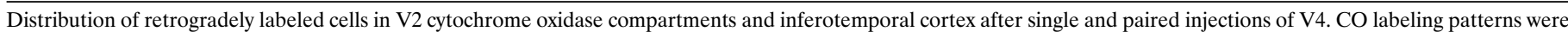

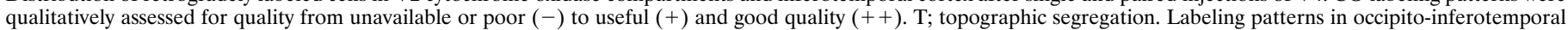
cortex were classified as C, clustered; D, diffuse; S, scattered; W, weak; DP, diffuse patchy; WC, weak clustered.

contains segregated regions of chrominance and luminance from thin stripes and contains segregated, orientation-specific regions from interstripe input. However, the results also indicate that specific V4 loci contain convergent inputs from V2 compartments. The observation that cells in V2 interstripes and cells in the pale portions of thin stripes can project specifically to single V4 loci indicates that convergence of functional streams can take place through cortico-cortical pathways rather than simply through intrinsic connectivity within each cortical area.

In the vast majority of cases, single injections of retrograde tracers in V4 labeled two or more sets of stripe compartments in $\mathrm{V} 2$. This is most clearly observed in case 6-BB, in which three sets of interstripe doublets were observed to span nearly $12 \mathrm{~mm}$ of opercular V2. This degree of convergence of V2 stripe-specific inputs to V4 is expected given the two- to threefold increase in receptive field size in V4 relative to V2 (Gattas et al., 1986).

\section{Organization of V4 projections to inferotemporal PITv}

\section{Overview}

The modular organization of outputs from V4 to PITv was analyzed in 10 hemispheres from 7 monkeys. Two different strategies were used to visualize the V4 projections to the temporal lobe. In seven cases, single (2) or paired (5) tracer injections were made into PITv in hemispheres that were sectioned in a horizontal plane. In three other cases, paired fluorescent tracer injections (1) or single WGA-HRP injections (2) of PITv were analyzed in tissue in which both the occipital operculum and contiguous anterior occipital (V4) and posterior temporal cortices were physically unfolded, flattened, and sectioned tangentially.

\section{Location of injection sites}

Single and paired retrograde tracers were injected into PITv under visual guidance using surface characteristics that included the posterior middle temporal, inferior occipital, and superior temporal sulci. Boussaoud et al. (1991) demonstrated that upper field portion of PITV (TEO) is generally located posterior to the PMTS, whereas the lower field is represented in a smaller region near the anterior portions of the PMTS. However, the PMTS is highly variable in its location and structure, making the localization of cortical areas relative to sulcal boundaries difficult, at best, given that areas may vary in location more than $5 \mathrm{~mm}$. Therefore, in most cases, we used the dense callosal band that is located at the representation of the superior vertical meridian at the anterior border of VOT to identify the border between occipital VOT with temporal lobe PITv.

\section{Segregated projections from V4 to PITv}

Injections of sensitive retrograde tracers into PITv produced dense labeling in V4, V4t, and VOT. Figure $4 E$ illustrates an unfolded cortical map that displays the PITv injection sites, the distribution of callosal-projecting neurons, and the distribution of retrogradely labeled cells in extrastriate visual cortex in case 11 . The injection sites were approximately $3 \mathrm{~mm}$ anterior to the callosal-free ring that contains the upper field representations of V4 and VOT. The diamidino injection site was at the crown of the superior temporal sulcus, near the border with PITd. The fast blue injection site was approximately $5 \mathrm{~mm}$ lateral (ventral) to the diamidino yellow injection site. Dense patches of retrogradely labeled cells were found in a large number of occipital and temporal lobe cortical areas, which are summarized in Table 2. Dense, interdigitating patterns of retrogradely labeled cells were found throughout a wide expanse of foveal and parafoveal upper and lower field V4. Labeled cells were found scattered throughout dorsal and ventral V2 and V3A, and a few cells were found in VP and perhaps V3. The laminar and horizontal distribution of labeled cells in V4 is illustrated in a stack of horizontal brain sections in Figure $4 A$. In these three $31 \mu \mathrm{m}$ thick brain sections spanning $500 \mu \mathrm{m}$ of V4, DY- and FB-labeled cells were found in multiple dense clusters that were aligned in both the supragranular and infragranular cortical layers. Individual clusters seemed to span 500-1000 $\mu \mathrm{m}$ and FB-labeled cell clusters were largely segregated from DY-labeled cell clusters.

The degree to which the labeled cell populations were segregated in the horizontal dimension was assessed using a SI (DeYoe et al., 1994). This index represents the proportion of cells in a 

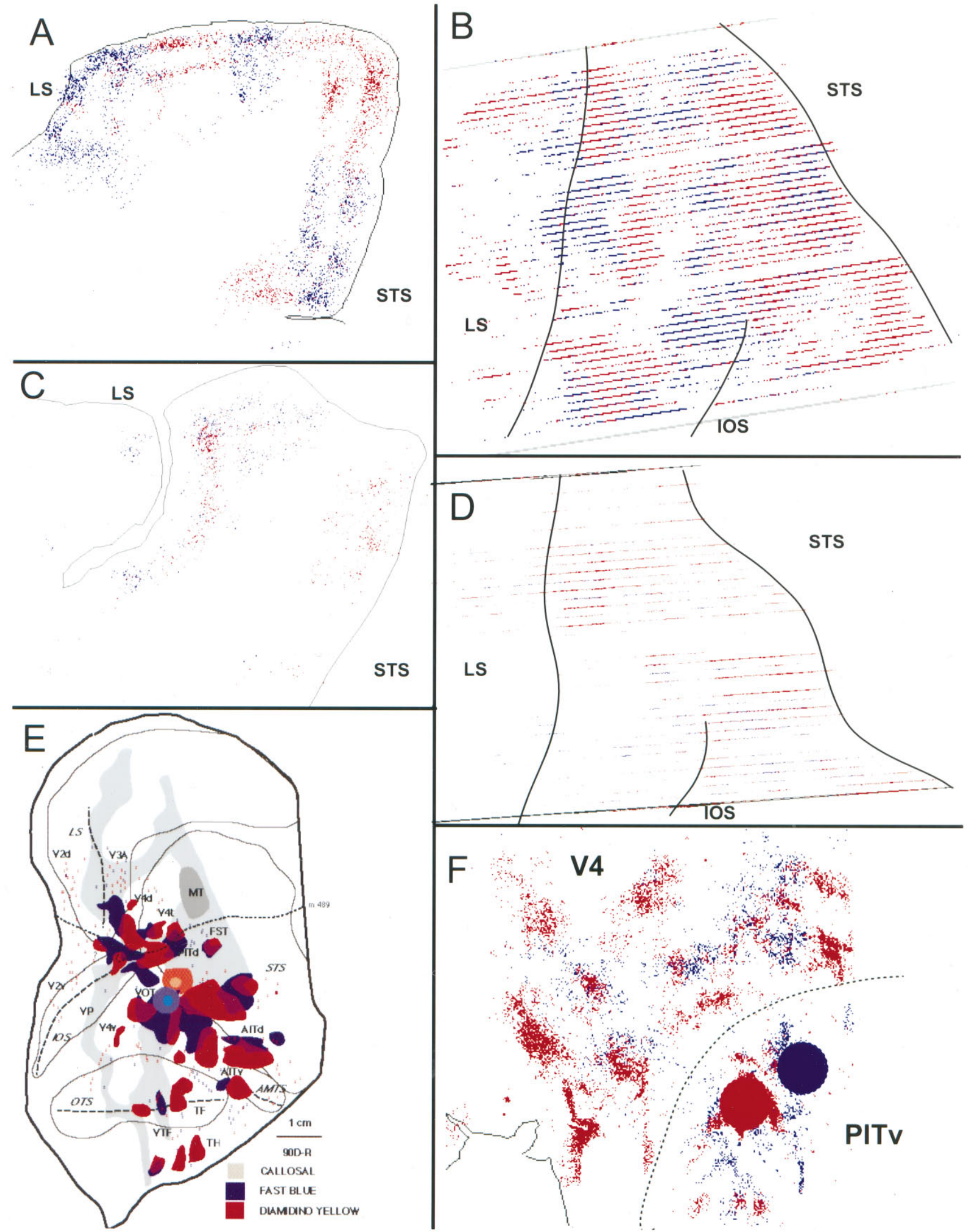

Figure 4. A. Two-dimensional map after dual fluorescent retrograde tracer injections of PITv (case 90D-R). B, Laminar and horizontal distribution of retrograde labeled cells in V4. Alternating clusters of BB- and NY-labeled cells in a series of three horizontal brain sections spanning $500 \mu \mathrm{m}$ through V4. Labeled cells are distributed in a bilaminar pattern, and like-labeled cells are segregated into multiple 1-2 mm wide clusters across the width of V4. Average SI for these sections $=0.80$ (case 90D-R). C, Segregated interdigitating labeling of V4. Three-dimensional (Figure legend continues) 


\begin{tabular}{|c|c|c|c|c|c|c|c|c|c|c|c|c|}
\hline $\begin{array}{l}\text { Case \# } \\
\text { tracer }\end{array}$ & Separation & $\mathrm{CC}$ & Myelin & $\mathrm{V} 2$ & V4 & $\mathrm{V} 4 \mathrm{t}$ & VOT & PITV & PITd & CITv & CITd & AITpv \\
\hline $10-\mathrm{FB}$ & $5 \mathrm{~mm}$ & + & + & $\mathrm{S}$ & Diffuse & + & + & I & + & + & + & + \\
\hline 10-ARG & & + & + & $\mathrm{S}$ & Cluster & + & + & I & + & + & - & $\mathrm{W}$ \\
\hline 11-FB & $5 \mathrm{~mm}$ & + & + & $\mathrm{S}$ & Segregated & - & + & I & + & + & + & + \\
\hline 11-DY & & + & + & $\mathrm{S}$ & Segregated & - & + & I & I & + & + & + \\
\hline $12-\mathrm{BB}$ & $5 \mathrm{~mm}$ & + & + & - & Cluster & - & + & I & + & + & + & + \\
\hline $12-\mathrm{NY}$ & & + & + & - & Diffuse & - & + & $\mathrm{I}$ & + & + & + & + \\
\hline 13-DY & Single & + & + & $\mathrm{S}$ & Cluster & + & + & I & + & + & + & - \\
\hline 14-BB & Single & + & + & & Cluster & $?$ & + & + & I & + & + & + \\
\hline $15-\mathrm{BB}$ & $3 \mathrm{~mm}$ & - & - & $\mathrm{S}$ & Segregated & $?$ & $\mathrm{~W}$ & I & $\mathrm{W}$ & + & $\mathrm{W}$ & + \\
\hline $15-N Y$ & & - & - & $\mathrm{S}$ & Cluster & - & $\mathrm{W}$ & I & + & + & + & + \\
\hline 16-FB & $4 \mathrm{~mm}$ & - & - & $\mathrm{S}$ & Overlap & - & + & I & + & I & + & + \\
\hline 16-DY & & - & - & $\mathrm{S}$ & Overlap & - & + & $\mathrm{I}$ & + & I & + & + \\
\hline 17-BB & $4 \mathrm{~mm}$ & + & $\mathrm{CO} / \mathrm{MT}$ & & Flat segregated & + & + & I & + & + & + & + \\
\hline $17-N Y$ & & & $\mathrm{CO} / \mathrm{MT}$ & & Flat segregated & + & + & I & + & + & + & $\mathrm{W}$ \\
\hline 4-WGA & Single & - & $\mathrm{CO} / \mathrm{MT}$ & - & Cluster & $?$ & + & I & + & + & + & $\mathrm{NE}$ \\
\hline 6-WGA & Single & - & $\mathrm{CO} / \mathrm{MT}$ & - & Cluster & - & - & I & - & + & + & $\mathrm{NE}$ \\
\hline
\end{tabular}

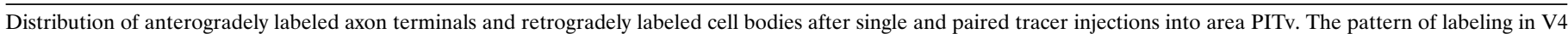

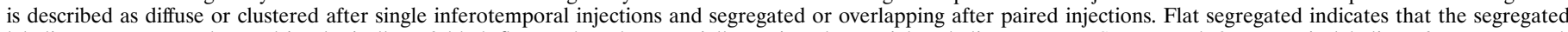

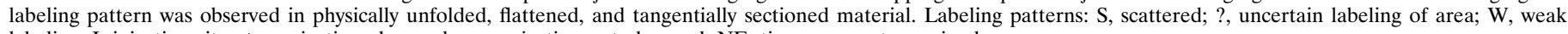
labeling; I, injection site; +, projection observed; -, projection not observed; NE, tissue was not examined.

given cluster labeled by one tracer as compared with the other tracer normalized for the total number of cell labeled by each tracer in the section or stack of sections. This index is calculated for each cluster of cells and an average index, corrected for the differential sensitivities of the tracers, is calculated. In this series of brain sections, the SI was 0.78 , indicating that clusters of DY-labeled cells generally contain less than $15 \%$ of the FBlabeled neurons. This index ranged from 0.5 to 1.16 for 8 clusters.

The overall distribution of the FB- and DY-labeled cells in V4 is illustrated in Figure $4 B$. This figure is an en face view of a threedimensional reconstruction of 80 horizontal brain sections spanning nearly $20 \mathrm{~mm}$ of the prelunate gyrus. The locations of the lunate, inferior occipital, and superior temporal sulci are projected onto this view for reference. DY- and FB-labeled cells are generally highly segregated. The pattern of labeling is complex, however, with some evidence of clusters extending dorso-ventrally across $2-3 \mathrm{~mm}$ of V4 and extending 1-3 $\mathrm{mm}$ antero-posteriorly. The dense pattern of labeled cells located near the anterior border of V4 at the STS is somewhat misleading in that this projection allows cells located deep within the STS to be visible on the cortical surface. This shortcoming was addressed by studying the projection of V4 to PITv in tissue that was physically unfolded and section tangential to the cortical surface (see below).

Figure $4 F$ illustrates an exemplary case of clustered labeling of/eb;n;j V4 after paired retrograde tracer injections of PITV (case 92-6R). The injection sites were located approximately 5 $\mathrm{mm}$ anterior to the callosal-free ring that defines V4-VOT in ventral occipital cortex. The nuclear yellow injection site was located approximately $4 \mathrm{~mm}$ lateral to the crown of the STS, and the BB injection site was located approximately $3 \mathrm{~mm}$ lateral and $2 \mathrm{~mm}$ anterior to the NY injection site. In this experiment, V4 and adjacent temporal lobe cortices were removed from the rest of the brain, and the superior temporal, inferior occipital, lunate, and portions of the occipito-temporal sulci were physically unfolded. The BB- and NY-labeled cells appeared in highly segregated clusters extending across a large portion of the width of foveal and parafoveal V4. In this case, approximately 15 dense clusters of NY-labeled cells were found across V4, and approximately 5 clusters of BB-labeled cells were found at intervening positions. The SI for these V4 clusters ranged from 0.27 to 1.59 with an average index of 0.73 . These values reflect both the high degree of segregation of cortical pathways and the diluting effect that unequal cell numbers causes this index (total BB-labeled cells = 2912; total NY-labeled cells $=10,420$ ). If the appropriate correction factor is really the total number of BB and NY cells in the brain rather than just V4, then these numbers are much closer and thus the SI will not be so adversely effected.

\footnotetext{
$\leftarrow$

reconstruction retrogradely labeled cells in V4 after separate injections of BB and NY in PITv. En face view of 80 horizontal brain sections through V4 (case 90D-R). $D$, Convergent labeling in three horizontal brain sections. BB- and NY-labeled cells are highly intermixed. Average SI for these three sections $=0.22$ (case 91-4R). E, Convergent labeling in a three-dimensional reconstruction of V4. NY-labeled cells are distributed widely across V4. BB-labeled cells are largely intermixed with the NY-labeled cells. Average $\mathrm{SI}=0.22$. More highly segregated clusters of BB-labeled cells are found in $1-2$ small patches in central portions of the map (case 91-4R). F, Segregated interdigitating labeling in tangentially sectioned occipito-temporal cortex. Occipito-temporal cortex was physically unfolded along the superior temporal, inferior occipital, and lunate sulci after the removal of opercular V1 and V2. Retrograde tracers were injected into PITV $5 \mathrm{~mm}$ anterior to the ventral callosal-free ring. HRP was applied to the cut ends of the corpus callosum to label callosal-projecting neurons that define the ventral callosal-free ring. Segregated clusters of BB- and NY-labeled cells were found across a wide expanse of V4 on the crown of the prelunate gyrus and lateral bank of the STS. NY-labeled cells were found in 15 1-2 mm wide clusters. Individual clusters were separated by approximately $2.5 \mathrm{~mm}$. Weaker BB labeling in V4 also was distributed in 5-6 irregularly shaped clusters. Average SI $=0.90$ (case 92-6R).
} 


\section{Divergent projections from V4 to PITv}

Whereas segregated patterns of labeling were often found in V4 after paired tracer injections of PITv, this was not always the case. It was hypothesized that the segregated projection patterns in V4 reflected discrete, nonoverlapping projections from clusters of cells in V4 to segregated domains in PITv. Because the PITV injections were placed at random, on occasion, individual injection sites should be expected to fall within similar domains and thus receive input from the same types of V4 compartments. However, such a pattern could also arise simply from topographic divergence in which a single site in V4 projects to two (or more) foci in PITv. Finally, an overlapping distribution of labeled cell populations in V4 could arise from injection sites that simply straddle to border between two distinct PITv modules.

The distribution of labeled cells from one such case is illustrated in Figure 4, $C$ and $D$. In this experiment, small injections of BB and NY were made into PITv 4-8 $\mathrm{mm}$ anterior to the callosal-free ring in ventral occipital cortex. The NY injection was located near the crown of the STS, and the BB injection was located $6 \mathrm{~mm}$ laterally on the inferior temporal gyrus. Retrogradely labeled cells were distributed across a wide expanse of extrastriate visual cortex extending from V4 to central portions of inferotemporal cortex. In V4, cells labeled by the BB and NY injections were largely intermixed. Figure $4 D$ illustrates the distribution of retrogradely labeled cells in three horizontal brain sections through V4 several millimeters ventral to the tip of the IOS. Whereas NY-labeled cells vastly outnumbered BB-labeled cells in this region, the average SI was 0.22 , indicating a highly convergent labeling of V4. The overall distribution of labeled cells in V4 is illustrated in Figure $4 E$. In this en face view of the prelunate gyrus, highly intermixed retrograde labeling is observed across approximately 80 brain sections spanning $15 \mathrm{~mm}$. Whereas NY-labeled cells outnumbered BB-labeled cells, the two cell populations were nearly always intermixed. However, in a few sections, less highly intermixed fields of BB-labeled cells were observed. These data indicate that a single focus in PITv may receive input from cells located in a wide expanse of V4. This widespread convergence onto PITv presumably reflects the increase in receptive field size and surround antagonism in PITV as compared with V4.

\section{DISCUSSION \\ What is V4?}

V4 has been identified by a number of investigators as a large cortical area, located on the prelunate gyrus and inferior occipital sulcus, that receives a major input from V2 and provides a major input to the temporal lobe. The possibility that V4 might consist of more than one subdivision has been raised by a number of investigators. On the basis of cortical connections, Zeki (1971) named the posterior portion of the V4 complex, V4, whereas the more anterior region on the crown of the prelunate gyrus was called V4a. Color selective cells were more frequently found in V4, as compared with V4a (Zeki, 1983). The idea that V4 consists of two subdivisions was further supported by electrophysiological mapping in awake monkeys, which provided variable evidence for two separate cortical maps on the prelunate gyrus (Baizer and Maguire, 1983). However, subsequent investigations have failed to confirm these electrophysiological results (for example, see Gattas et al., 1988). Instead, a single V4 was described extending from a vertical meridian representation at the posterior border and a representation of the horizontal meridian at its anterior border with V4t.

The pattern of interhemispheric connections to this region of cortex is best described at two dense callosal bands surrounding a callosal-free zone. This pattern suggests the presence of two, mirror symmetrical maps that adjoin along the representation of the horizontal meridian. Electrophysiological mapping in ventral cortex (Felleman et al., 1986) has revealed two mirror symmetrical representations of the upper quadrant in a region that Gattas et al. reported to contain only a single map. The posterior field corresponds to V4 proper, whereas the anterior area was named the VOT (Felleman et al., 1986; Van Essen et al., 1990).

In the current experiments, injections of V4 were confined to the crown of the prelunate and thus to foveal and parafoveal representations of the lower visual field. These injections were considered to be contained within V4 because of their dense feedforward labeling of V2, widespread projections to the temporal lobe, and projections to a belt of cortex anterior to the injection site but far posterior to the PMTS. In ventral cortex, this projection field corresponds to VOT, whereas a similar band is observed in dorsal cortex to extend into the STS from near the tip of the IOS. This region of cortex is tentatively identified as DOT to indicate its potential relationship to VOT, but to also to retain the distinction until more information is available.

Studies of the cortical connections of DL, the presumed V4 homolog, in owl monkey and squirrel monkey indicate that DL consists of two separate areas, DL rostral (DLr) and DL caudal (DLc), that differ in the strengths of their connections with V2 and parietal cortex. (Cusick et al., 1988; Steele et al.,1991; Weller et al., 1991). Similar observations have been made by Stepniewska and Kaas (1996) in macaque monkeys. According to this view, V4 is homologous with DLc, whereas VOT and DOT would be contained within DLr.

\section{Clustering of functional properties in V4}

Physiological investigations of receptive field properties of neurons in V4 have consistently found a high incidence of orientation and chromatic selectivity but not necessarily in the same neurons (Desimone and Schein, 1987). Using electrophysiological methods, DeYoe et al. (1992) found evidence for functional clustering with respect to both color and spatial frequency selectivity, and Gallant et al. (1993) reported clustering with respect to Cartesian and non-Cartesian grating selectivities. The most recent evidence for functional clustering or modularity within V4 comes from functional imaging studies using optical recording of intrinsic cortical signals. Ghose et al. (1994a,b, 1995) have demonstrated small foci in V4 that were preferentially activated by chromatic, nonoriented gratings or oriented, luminance gratings. However, the majority of V4 sites were activated by more complex stimuli, perhaps consisting of both chromatic and orientation components. Finally, in preliminary studies using stimuli designed to stimulate specific color opponent mechanisms, Ghose et al. (1995) observed an organized representation of color space within the chromatic selective modules of V4. Overall, these data indicate that V4 contains a wide range of receptive field types, many of which seem to be clustered together. However, the relationship between these functional domains and the pattern of V2 inputs remains to be determined.

\section{Organization of V2 inputs to V4}

Overview of the modular organization of $V 2$

V2 contains elongated, stripe-like regions that differ in their staining for the metabolic enzyme cytochrome oxidase, patterns of inputs from V1, inputs from the pulvinar, outputs to contralateral V2, and outputs to ipsilateral extrastriate cortical areas. Dense 
cytochrome oxidase thin stripes are distinguished from thick stripes by their inputs from V1 cytochrome oxidase blobs (Livingstone and Hubel, 1984) and layer 4B, respectively (Livingstone and Hubel, 1987). Furthermore, thick stripes provide a major input to MT (DeYoe and Van Essen, 1985; Shipp and Zeki, 1985) and V3 (Felleman et al., 1988). Both thick and thin stripes receive direct input from the inferior pulvinar (Livingstone and Hubel, 1986?) and project to contralateral V2, whereas the cytochrome oxidase pale interstripe do not (Olavarria and Abel, 1996). In contrast, cells in the thin stripes and interstripes project densely to V4, V3a, and V4t (DeYoe and Van Essen, 1985; Shipp and Zeki, 1985; Felleman et al., 1988; Nakamura et al., 1994).

\section{Segregated thin stripe and interstripe inputs to V4}

The current results confirm and extend previous reports of the specificity of visual pathways linking V2 with V4, which indicated that cells in V2 cytochrome oxidase thin stripe and interstripe compartments project to largely segregated zones within V4 (DeYoe et al., 1988, 1994; Shipp and Zeki, 1989). The diversity of projection patterns observed in the current experiments, in conjunction with those previously published reports, indicates that V4 contains at least three compartments as inferred by the pattern of inputs they receive from V2.

Figure 5 illustrates schematically the patterns of feedforward inputs from V2 to V4 that can be inferred from the current data. In agreement with DeYoe et al. $(1988,1994)$, we found in several cases V4 injections that labeled either the thin stripe or interstripe compartment. In the cases with paired injections, separations of 3-4 $\mathrm{mm}$ in V4 could label cells in separate but topographically overlapping cytochrome oxidase compartments. Thus, injections at the tips of the red and gray arrows or at the green and gray arrows in Figure 5 would be expected to label segregated cell populations in the thin and interstripes, respectively. Experiments such as these argue for V4 regions receiving V2 thin stripe or interstripe afferents exclusively.

\section{Heterogeneity within V2 stripe compartments}

In the current experiments, on occasion, pairs of retrograde tracer injections in V4 labeled different clusters of cells within the same $\mathrm{V} 2$ thin stripe. Because the injections were placed in nonoverlapping foci, separated by $\sim 3 \mathrm{~mm}$, the interdigitating pattern of labeled cells observed in V2 implies that V4 contains at least two different fields that receive input from different cell clusters in V2 thin stripes (see Fig. 5). Recently, Roe and Ts'o (1995) demonstrated a submodular organization within V2 thin stripes such that $\sim 500-1000 \mu \mathrm{m}$ wide zones are preferentially activated by isoluminant color or luminance stimuli, respectively. Because the cells within the cytochrome oxidase blobs of V1 are functionally heterogeneous (cf. Edwards et al., 1995) and V1 blobs project to V2 thin stripes (Livingstone and Hubel, 1984), it is tempting to speculate that the two clustered arrays of cells found in V2 thin stripes reflect the double role of the Blob-dominated system. Perhaps, at the level of V2 thin stripes, the brightness properties of blobs (and their magnocellular input for added dynamic range) become segregated from the color-specific (dual opponent receptive fields) properties of blobs. Functional subcompartments within V2 thin stripes thus would represent, explicitly, the dual properties of the Blob system that remain "multiplexed" in V1.

\section{Integration across stripe compartments}

In two experimental hemispheres, single V4 injections labeled clusters of V2 cells that extended from within a thin stripe to an adjacent interstripe region. In one case, the thin stripe was clearly composed of cytochrome oxidase dense clusters surrounded by pale regions. Furthermore, the labeled cells were largely confined to the cytochrome oxidase-pale zones of these thin stripes. This continuous mapping of inputs from two adjacent stripe compartments to a single V4 injection site suggests that V4 contains zones of convergence of multiple V2 stripe subcompartments. V2 thin stripes are functionally heterogeneous, consisting of distinct clusters of chromatic and luminance selective neurons (Roe and Ts'o, 1995). Although it is unclear whether this heterogeneity is closely associated with differences in cytochrome oxidase activity, it is a testable hypothesis that the cytochrome oxidase dense zones of V2 thin stripes contain chromatic selective, nonoriented neurons, whereas the pale regions of thin stripes contain the luminance selective clusters.

The clustered projection pattern within the V2 interstripe regions is suggestive of the mapping of two different stimulus properties or dimensions from V2 to V4. Because V2 interstripes contain orientation-selective cells, this clustered segregation might simply reflect the projections of cells with different orientation specificities to segregated regions of V4. Alternatively, this segregation might reflect the differential processing of Cartesian and non-Cartesian stimuli (Gallant et al., 1993), or might reflect differential processing of illusory contours and occluded objects as compared with simpler texture processing (Peterhans and van der Heydt, 1994).

\section{Organization of V4 outputs to inferotemporal PITv}

Single foci in inferotemporal PITv receive input from multiple clusters of cells distributed across many millimeters of V4. Large injections tend to receive input from a homogeneous expanse of $\mathrm{V} 4$, whereas smaller injections $(\sim 250 \mathrm{nl})$ tend to receive input from multiple $1-2 \mathrm{~mm}$ wide clusters that are separated by $2-3 \mathrm{~mm}$. This high degree of convergence is thought to be necessary to account for the increase in receptive field size from V4 to PITV (Boussaoud et al., 1991; Gattas et al., 1988). Alternatively, strong topographic convergence of V4 inputs to PITv might provide the required substrate necessary for shifts of visual attention across the visual scene (Olshausen et al., 1993).

Paired injections of distinguishable retrograde tracers placed 3-4 mm apart in PITv often labeled segregated yet interdigitating populations in the upper layers of V4 (see Fig. 5). Single PITv foci receive convergent input from multiple, irregularly shaped V4 output "modules." Whereas single retrograde injections of PITV cannot identify the number of terminal zones achieved by each V4 module, retrograde injections of V4 suggest that more than one PITv focus provides feedback to each V4 site. Thus, Figure 5 represents only one such set of terminal fields and does not reflect the divergence of the projections from V4 to PITv.

Does the modular output pattern from V4 simply reflect the pattern of inputs that they receive from V2, or does this output array represent a completely unrelated set of functional parameters? At the present time there is not clear answer to this problem, but there are several observations that bear directly on this issue. First, in the DeYoe et al. (1994) study and in the current results, V4 injections that label separate thin stripe and interstripe compartments of V2 tend to label segregated loci in PITv. Conversely, paired injections that produce overlapping label in V2 compartments yield overlapping projection fields in PITv. Second, the modular output array of V4 seems to consist of units that are spaced roughly 2-3 mm apart. This spacing is in rough agreement with the size of axonal terminations in V4 after anterograde injections of V2 (Rockland, 1992; Xiao 
Figure 5. Modular connections in early and intermediate visual processing. Schematic representation of the organization of V2, V4, and PITv and their major connections. V2 thin stripes are functionally heterogeneous with segregated clusters selective for chromatic or luminance processing. V2 interstripes are also functionally heterogeneous with portions selective for stimulus orientation. Two or more thin stripes or two to three sets of interstripes project in a convergent manner to single loci in V4. Clustered subregions within V2 thin stripes project to segregated regions in V4 that may reflect different aspects of surface processing such as luminance and chromatic analysis. Similarly, repeating clusters within interstripes project to single foci in V4, whereas intervening foci project to nearby locations within V4. This pattern may reflect orientation-specific projections from V2 to V4 or some submodality within interstripes. Feedforward projections from V4 to two foci separated by $\sim 4 \mathrm{~mm}$ in PITv are distributed in segregated yet interdigitating arrays across the prelunate gyrus. Single PITv foci may receive input from an array of V4 modules that are spaced roughly $2.5 \mathrm{~mm}$ apart. A high degree of convergence characterized this projection from $\mathrm{V} 4$ to

PITv

Layer 4

V4

Layer 2/3

\section{V4 \\ Layer 4}
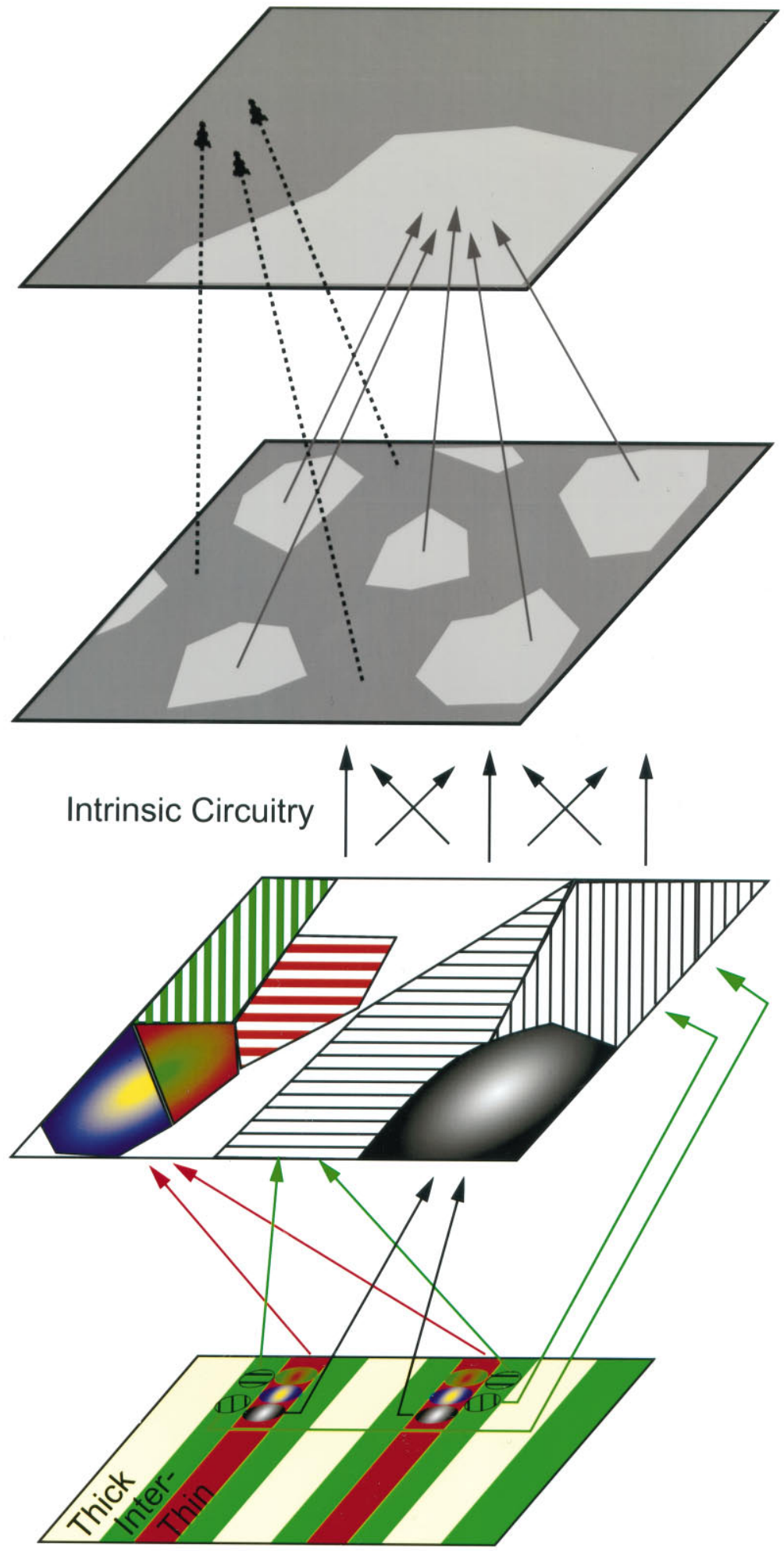

and Felleman, 1996). Finally, small foci in V2 thin stripes and interstripes project to major foci in $\mathrm{V} 4$ that are roughly 2-3 $\mathrm{mm}$ apart (Xiao and Felleman, 1996).

\section{Functional organization of PITv}

Two lines of evidence suggest that inferotemporal PITv contains a modular organization of extrinsic cortical connections. First, in cases of V4 injections that labeled segregated thin stripe and interstripe portions of V2, the feedback projections from PITv were also segregated into multiple clusters approximately $3-6 \mathrm{~mm}$ in width (DeYoe et al., 1994). Second, the feedforward projections from V4 to inferotemporal cortex often arise from cell clusters separated by 2-3 $\mathrm{mm}$ that terminate in segregated zones in PITv separated by $\sim 4$ mm (Felleman et al., 1992; DeYoe et al., 1994).

Functional evidence for a modular organization within posterior inferotemporal cortex comes from recent physiological stud- 
ies of shape and color selectivity. Tanaka and colleagues found that cells with complex receptive field properties are found in V4, and in posterior and anterior IT (Kobatake and Tanaka, 1994; Tanaka et al., 1986, 1991). In PITv, these cells are numerous (up to $49 \%$ ) and are intermixed with cells with simple component features. However, cells that are selective for specific simple or "critical" features, including color and orientation, were found clustered in PITv. Recent electrophysiological (Fujita et al., 1992) and optical recording (Wang et al., 1994) experiments suggest that anterior IT consists of columns of neurons preferring similar features or objects. It is tempting to speculate that the complex properties of cortical columns in anterior IT are built up through the specific columnar projections from more simple columns in PITv (Saleem et al., 1993).

\section{REFERENCES}

Baizer JS, Ungerleider LG, Desimone R (1991) Organization of visual inputs to the inferior temporal and posterior parietal cortex in macaque. J Neurosci 11:168-190.

Blasdel G, Livingstone M, Hubel D (1993) Optical imaging of orientation and binocularity in visual areas 1 and 2 of squirrel monkey (Samiri sciureus) cortex. Soc Neurosci Abstr 19:1500.

Boussaoud D, Desimone R, Ungerleider LG (1991) Visual topography of area TEO in the macaque. J Comp Neurol 306:554-575.

Carroll EW, Wong-Riley MTT (1984) Quantitative light and electron microscopic analysis of cytochrome oxidase-rich zones in the striate cortex of the squirrel monkey. J Comp Neurol 222:1-17.

Cowan WM, Gottlieb DI, Hendrickson AE, Price JL, Woolsey TA (1972) The autoradiographic demonstration of axonal connections in the central nervous system. Brain Res 37:21-51.

Cusick CG, Kaas JH (1988) Cortical connections of area 18 and dorsolateral visual cortex in squirrel monkeys. Vis Neurosci 1:211-237.

Desimone R, Schein SJ (1987) Visual properties of neurons in area V4 of the macaque: sensitivity to stimulus form. J Neurophysiol 57:835-868.

DeYoe EA, Van Essen DC (1985) Segregation of efferent connections and receptive field properties in visual area V2 of the macaque. Nature 317:58-61.

DeYoe EA, Felleman DJ, Knierim JJ, Olavarria J, Van Essen DC (1988) Heterogeneous subregions of macaque visual area V4 receive selective projections from V2 thin-stripe and interstripe subregions. Invest Ophthamol Vis Sci [Suppl] 29:115.

DeYoe EA, Glickman S, Wieser J (1992) Clustering of visual response properties in cortical area V4 of macaque monkeys. Soc Neurosci Abstr 18:592.

DeYoe EA, Felleman DJ, Van Essen DC, McClendon E (1994) Multiple processing streams in occipito-temporal visual cortex. Nature 371:151-154.

Distler C, Boussaoud D, Desimone R, Ungerleider LG (1993) Cortical connections of inferior temporal area TEO in macaque monkeys. J Comp Neurol 334:125-150.

Edwards DP, Purpura KP, Kaplan E (1995) Contrast sensitivity and spatial frequency responses of primate cortical neurons in and around cytochrome oxidase blobs. Vision Res 35:1501-1523.

Felleman DJ, Burkhalter A, Van Essen DC (1996) Cortical connections of areas V3 and VP of macaque visual cortex. J Comp Neurol, in press.

Felleman DJ, DeYoe EA, Knierim JJ, Olavarria J, Van Essen DC (1988) Compartmental organization of projections from V2 to extrastriate areas V3, V3A, and V4t in macaque monkeys. Invest Ophthamol Vis Sci [Suppl] 29:115.

Felleman DJ, McClendon E (1991a) Cortical connections of posterior inferotemporal cortex of macaque monkeys. Invest Ophthamol Vis Sci [Suppl] 32:1810.

Felleman DJ, McClendon E (1991b) Modular connections between V4 and temporal lobe area PITv in macaque monkeys. Soc Neurosci Abstr 17:1282.

Felleman DJ, McClendon E, Lin K (1992) Modular segregation of visual pathways in occipital and temporal lobe visual areas in the macaque monkey. Soc Neurosci Abstr 18:390.

Fujita I, Tanaka K, Ito M, Cheng, K (1992) Columns for visual features of objects in monkey inferotemporal cortex. Nature 360:343-346.

Gallant JL, Braun J, Van Essen DC (1993) Selectivity for polar, hyper- bolic, and Cartesian gratings in macaque visual cortex. Science 259:100-103.

Gallyas F (1979) Silver staining of myelin by means of physical development. Neurol Res 1:203-209.

Ghose GM, Roe AW, Ts'o DY (1994) Features of functional organization within primate V4. Soc Neurosci Abstr 20:840.

Ghose GM, Shey J, Ts'o DY (1994) Functional organization of V4 as revealed by in vivo optical recording. Invest Ophthamol Vis Sci [Suppl] 35:1663.

Ghose GM, Ts'o DY (1995) Orientation and color segregation in primate V4. Soc Neurosci Abstr 21:15.4.

Hubel DH, Livingstone MS (1987) Segregation of form, color, and stereopsis in primate area 18 . $\mathrm{J}$ Neurosci 7:3378-3415.

Kobatake E, Tanaka K (1994) Neuronal selectivities to complex object features in the ventral visual pathway of the macaque cerebral cortex. J Neurophysiol 71:856-867.

Lachica EA, Beck PD, Casagrande VA (1992) Parallel pathways in macaque monkey striate cortex: anatomically defined columns in layer III. Proc Natl Acad Sci USA 89:3566-3570.

Levitt JB, Kiper DC, Movshon JA (1994) Receptive fields and functional architecture of macaque V2. J Neurophysiol 71:2517-2542.

Livingstone MS, Hubel DH (1984) Anatomy and physiology of a color system in the primate visual cortex. J Neurosci 4:309-356.

Livingstone M, Hubel DH (1987) Connections between layer 4B of area 17 and the thick cytochrome oxidase stripes of area 18 in the squirrel monkey. J Neurosci 7:3371-3377.

Malach R, Tootell RBH, Malonek D (1994) Relationship between orientation domains, cytochrome oxidase stripes, and intrinsic horizontal connections in squirrel monkey. Cereb Cortex 4:151-165.

Mesulam MM (1978) Tetramethylbenzidine for horseradish peroxidase histochemistry: a non-carcinogenic blue reaction product with superior sensitivity for visualizing neural afferents and efferents. J Histochem Cytochem 26:106-117.

Morel A, Bullier J (1990) Anatomical segregation of two cortical visual pathways in the macaque monkey. Vis Neurosci 4:555-578.

Nakamura H, Gattass R, Desimone R, Ungerleider LG (1993) The modular organization of projections from areas V1 and V2 to areas V4 and TEO in macaques. J Neurosci 13:3681-3691.

Olavarria J, Abel PL (1996) The distribution of callosal connections correlated with the pattern of cytochrome oxidase stripes in visual area V2 of macaque monkeys. Cereb Cortex 6:631-639.

Olavarria J, van Sluyters RC (1985) Unfolding and flattening the cortex of gyrencephalic brains. J Neurosci Methods 15:191-202.

Peterhans E, von der Heydt R (1993) Functional organization of area V2 in the alert monkey. Eur J Neurosci 5:509-524.

Rockland KS (1992) Configuration, in serial reconstruction, of individual axons projecting from area V2 to V4 in the macaque monkey. Cereb Cortex 2:353-374.

Roe AW, Ts'o DY (1995) Visual topography in primate V2: multiple representation across functional stripes. J Neurosci 15:3689-3715.

Saleem K, Tanaka K, Rockland KS (1993) Specific and columnar projection from area TEO to TE in the macaque inferotemporal cortex. Cereb Cortex 3:454-464.

Sereno MI, McDonald CT, Allman JM (1994) Analysis of retinotopic maps in extrastriate cortex. Cereb Cortex 6:601-620.

Shipp S, Zeki S (1985) Segregation of pathways leading from V2 to areas V4 and V5 of macaque monkey visual cortex. Nature 315:322-325.

Shipp S, Zeki S (1995) Segregation and convergence of specialized pathways in macaque monkey visual cortex. J Anat 187:547-562.

Steele GE, Weller RE, Cusick CG (1991) Cortical connections of the caudal subdivision of the dorsolateral area (V4) in monkeys. J Comp Neurol 306:495-520.

Stepniewska I, Kaas JH (1996) Topographic patterns of V2 cortical connections in macaque monkeys. J Comp Neurol, in press.

Tanaka K, Weber H, Creutzfeldt OD (1986) Visual properties and spatial distribution of neurons in the visual association cortex of the prelunate gyrus of the awake monkey. Exp Brain Res 65:11-37.

Tanaka K, Saito H-A, Fukada Y, Moriya M (1991) Coding of visual images of objects in the inferotemporal cortex of the macaque monkey. J Neurophysiol 66:170-189.

Tootell RBH, Silverman MS (1985) Two methods for flat-mounting cortical tissue. J Neurosci Methods 15:177-190.

Tootell RBH, Born RT, Ash-Bernal R (1993) Columnar organization in visual cortex in non-human primates and man. In: Functional organi- 
zation of visual cortex in man (Gulyas B, Ottoson D, Roland PE, eds), pp 59-74. Oxford: Pergamon.

Ts'o DY, Frostig RD, Lieke EE, Grindvald A (1990) Functional organization of primate visual cortex revealed by high resolution optical imaging. Science 249:417-420.

Van Essen DC, Gallant JL (1994) Neural mechanisms of form and motion processing in the primate visual system. Neuron 13:1-10.

Van Essen DC, Felleman DJ, DeYoe EA, Olavarria J, Knierim J (1990) Modular and hierarchical organization of extrastriate visual cortex in the macaque. Cold Spring Harbor Symp Quant Biol 55:679-696.

Wang G, Tanaka K, Tanifuji M (1994) Optical imaging of functional organization in macaque inferotemporal cortex. Soc Neurosci Abstr 20:31.

Weller RE, Kaas JH (1985) Cortical projections of the dorsolateral visual area in owl monkeys: the prestriate relay to inferior temporal cortex. J Comp Neurol 234:35-59.

Weller RE, Steele GE, Cusick CG (1991) Cortical connections of dorsal cortex rostral to VII in squirrel monkeys. J Comp Neurol 306:521-537.

Wong-Riley MTT, Carroll EW (1984) Quantitative light and electron microscopic analysis of cytochrome oxidase-rich zones in VII prestriate cortex of the squirrel monkey. J Comp Neurol 222:18-37.

Xiao Y, Felleman DJ (1995) Functional imaging and cortical connections of V2 thin and interstripe compartments. Soc Neurosci Abstr 21:1275.

Xiao Y, Felleman DJ (1996) Modular organization of axonal projections from functionally identified V2 compartments to V4 in macaques. Soc Neurosci Abstr 22:398.

Yoshioka T, Levitt JB, Lund JS (1992) Intrinsic lattice connections of macaque monkey visual cortical area V4. J Neurosci 2:2785-2802.

Zeki S, Shipp S (1989) Modular connections between areas V2 and V4 of macaque monkey visual cortex. Eur J Neurosci 1:494-506. 